\title{
Energetics of the Deep Gulf of Mexico
}

\author{
Aljaz MASLO \\ Centro de Investigación Científica y Educación Superior de Ensenada, Ensenada, Baja California, Mexico \\ JoÃo MARCos AZEvedo Correia de SOUZA \\ MetOcean Solutions, New Plymouth, New Zealand \\ JULIO SHEINBAUM PARDO \\ Centro de Investigación Científica y Educación Superior de Ensenada, Ensenada, Baja California, Mexico
}

(Manuscript received 18 December 2019, in final form 2 March 2020)

\begin{abstract}
The evaluation of the ocean energy balance is crucial for improving the fundamental understanding of the mechanisms sustaining ocean circulation. Based on the outputs of the ROMS ocean model, the energy cycle, eddy-mean flow interactions, and energy pathways of the deep Gulf of Mexico (GoM) have been investigated in this study. The theoretical framework for the analysis is based on the energy equations for the time-mean and time-varying flow, where some of the terms were split into their horizontal and vertical components to monitor the energy pathways. Of the energy maintaining deep kinetic energy (KE), approximately $75 \%$ is transferred from the upper layer to the deep layer by vertical pressure work (PW), about $6 \%$ by the horizontal PW through the Yucatan and Florida straits, and $\sim 19 \%$ is generated through the processes related to baroclinic instabilities. The mean circulation generates eddies in the upper layer, while eddies drive mean circulation in the deep layer. Energy is transferred downward in the eastern and western part of the Gulf, upward in the deep western-central part, and a strong westward energy transport can be observed below 2000-m depth.
\end{abstract}

\section{Introduction}

Energy balance analysis is useful for advancing our current understanding of the mechanisms maintaining the ocean currents. This balance is closely tied to the nature of the interaction between eddies and the mean flow because of the role of eddies in redistributing momentum and heat in the ocean (Flury and Rummel 2007). Eddies can transport energy to distant regions of the basin, interact with the mean flow, and finally dissipate much of the energy into the bottom and lateral friction (Holland et al. 1983).

Eddies play a central role in ocean dynamics, and the kinetic energy of eddies is usually an order of magnitude larger than that of the mean circulation (Ferrari and Wunsch 2009). Accordingly, most energy analysis studies focus on understanding the eddy kinetic energy properties of the flow. For instance, Yang et al. (2013) studied the eddy energy sources and sinks of the South

Corresponding author: Aljaz Maslo, maslo@cicese.mx
China Sea (SCS). Their energy budget analysis suggests that the most important eddy energy source for the SCS is the energy released from the available potential energy, followed by the energy convergence from surrounding areas in a horizontal direction and energy released from barotropic instabilities. Zhan et al. (2016) examined the sources, sinks, and redistribution of eddy kinetic energy in the Red Sea. They conclude that the eddies acquire kinetic energy predominantly from the conversion of eddy available potential energy, followed by the transfer from the mean kinetic energy and from the direct generation due to time-varying wind stress. The eddy energy sources in the Sea of Okhotsk are investigated in the work of Stepanov (2018). He argues that the contribution of baroclinic instabilities predominates over that of barotropic instabilities in the generation of mesoscale variability along the western boundary of the Sea of Okhotsk. The work of Zhai and Marshall (2013) is particularly interesting because it focuses on the vertical fluxes of eddy energy in the North Atlantic subtropical and subpolar gyres and studying such fluxes in the 
Gulf of Mexico (GoM) is the primary motivation for this article. Zhai and Marshall (2013) concluded that the vertical energy flux is downward in the subtropical gyre and upward in the subpolar gyre to reconcile the mismatch between the depth of eddy energy sources related to baroclinic instabilities and the vertical structure of the horizontal dispersion of eddy energy. However, the work of Zhai and Marshall (2013) was limited to exploring the eddy kinetic energy generation and energy fluxes and did not take into consideration the exploration of the whole energy cycle, thereby including in their analysis the generation, dissipation, conversion, and pathways of the mean kinetic energy, mean available potential energy, and eddy available potential energy reservoirs. Kang and Curchitser (2015, hereafter KC15) derived the energy equations to analyze the energy exchange cycle of the Gulf Stream and concluded that eddy kinetic energy in the region is generated predominantly by the barotropic instability followed by the baroclinic instability. A quantitative description of the deep GoM energy cycle is crucial for improving the current understanding of how the deep Gulf general circulation functions. The energy equations presented by $\mathrm{KC} 15$ consist of a theoretical framework for the evaluation of the deep GoM energy cycle and provides us with a means of establishing a broader physical picture of the processes involved in maintaining the deep circulation.

The GoM forms a semienclosed region with connections to the Caribbean Sea through the Yucatan Channel and to the Atlantic Ocean through the Florida strait and is often approximated as a two-layer system where the boundary between the upper and the deeper layer is usually situated at 1000-m depth (Hamilton 2009; Cardona and Bracco 2016; Chang and Oey 2011). The upper-layer circulation is dominated by the Loop Current (LC), and is covered fairly well in the literature (Alvera-Azcárate et al. 2009; Hamilton et al. 1999; Oey et al. 2005), while the deep layer has attracted less attention because it is less easily observed. Numerical studies present a general cyclonic mean flow of the deep Gulf (Oey and Lee 2002; Lee and Mellor 2003; Chang and Oey 2011; Maslo et al. 2020), which has been supported by observations (Weatherly et al. 2005; DeHaan and Sturges 2005; Pérez-Brunius et al. 2018). Observations indicate that the deep GoM is a surprisingly energetic environment, where the recorded speeds in the northern Gulf can exceed $90 \mathrm{~cm} \mathrm{~s}^{-1}$ (Hamilton and LugoFernandez 2001). Recently, Pérez-Brunius et al. (2018) analyzed 158 drifting float trajectories, the largest deep GoM float study thus far, and presented the mean velocity vector field. The mean deep GoM circulation deduced from the subsurface floats consists of two mean flow structures: a cyclonic boundary current and a deep cyclonic gyre in the western part of the Gulf. The same mean flow structures were also simulated reasonably well by Maslo et al. (2020) using a 5-yr (2010-14) ROMS numerical simulation to study the connectivity of the deep Gulf, and the same numerical dataset is employed in this study.

The studies exploring the sources of energy in the deep GoM and the interaction between the upper and lower layer are mostly concentrated in the eastern Gulf due to high interest in understanding the dynamics of the LC and the mechanism related to the detachments of its anticyclonic eddies. Oey and Lee (2002) simulation results show that the source of deep eddy kinetic energy east of approximately $91^{\circ} \mathrm{W}$ comes from the LC while over the western Gulf additional source is from the southwestward propagating Loop Current eddies (LCEs). Lee and Mellor (2003) suggested, based on their model results, that the deep cyclonic circulation in the eastern Gulf, driven by the LC fluctuations of the upper layer, plays an important role as an energy source for the whole deep Gulf circulation. Oey (2008) analyzed the results of a high-resolution numerical model to explain the origin of the deep eddies and their important role in forcing the topographic Rossby waves that disperse energy at the northern slopes of the Gulf. Hamilton et al. (2016) presented an overview of an observational study of the LC and reported that the lower layer proved to be highly energetic only during relatively short periods of time (2-3 months), just prior to or during the eddy detachment due to baroclinic instability. Donohue et al. (2016) observed a marked increase of deep eddy kinetic energy during the LCEs detachment and formation events. Moreover, they reported that the deep eddies that occur during these events gain their high-energy levels in a pattern consistent with developing baroclinic instability. However, little is known about the processes and pathways by which energy is transported through the rest of the Gulf. Comprehending the physics of these processes is fundamental for improving the understanding and prediction of the deep-water environment that impacts oil and gas operations, as well as biological, sediment, and pollutant transport.

This paper focuses on the deep Gulf energy cycle, eddy-mean flow interaction, and energy pathways based on a 5-yr (2010-14) ROMS numerical simulation. In particular, we aim to answer the following research questions: 1) How is the energy driving the deep GoM circulation maintained? 2) What are the characteristics of the eddy-mean flow interaction in the deep GoM? 3) How and where is the energy transferred from the upper layer to the deeper layer?

The paper is organized as follows: section 2 describes the energy equations and a numerical simulation employed in this study, section 3 presents the main results about the energetics of the deep GoM, and section 4 summarizes the paper. 


\section{Methodology}

\section{a. Energy budget equations}

The derivation of the energy equations follows the work of $\mathrm{KC} 15$ and is based on the Reynolds-averaged Navier-Stokes equations, along with the continuity and density equation, which are given by using Boussinesq approximation:

$$
\begin{aligned}
\frac{\partial u}{\partial t}+\mathbf{u} \cdot \nabla u-f v & =-\frac{1}{\rho_{0}} \frac{\partial p}{\partial x}+\mathscr{F}_{u}+\mathscr{D}_{u}, \\
\frac{\partial v}{\partial t}+\mathbf{u} \cdot \nabla v+f u & =-\frac{1}{\rho_{0}} \frac{\partial p}{\partial y}+\mathscr{F}_{v}+\mathscr{D}_{v}, \\
\frac{\partial p}{\partial z} & =-\rho g, \\
\nabla \cdot \mathbf{u} & =0, \\
\rho(x, y, z, t) & =\rho_{r}(z)+\rho_{a}(x, y, z, t), p(x, y, z, t) \\
& =p_{r}(z)+p_{a}(x, y, z, t), \quad \text { and } \\
\frac{\partial \rho_{a}}{\partial t}+\mathbf{u} \cdot \nabla \rho_{a} & =\frac{\rho_{0} N^{2}}{g} w+\mathscr{S}_{\rho}+\mathscr{D}_{p} .
\end{aligned}
$$

Here $\mathbf{u}=(u, v, w)$ is the velocity vector, $f$ is the Coriolis frequency, $\rho$ is density with $\rho_{0}=1000 \mathrm{~kg} \mathrm{~m}^{-3}$ its constant value, $p$ is pressure, $\mathscr{F}$ and $\mathscr{D}$ represent terms of forcing and turbulent diffusivity, respectively, $\mathscr{S}$ is a density source term, and $g$ is the gravitational acceleration. The density and pressure are given in Eq. (5), where $\rho_{r}$ and $p_{r}$ are the reference density and pressure, and $\rho_{a}$ and $p_{a}$ represent the perturbation density and pressure. The reference density and reference pressure were chosen to be the time mean and area mean values that are constant at a given depth. The density transport in Eq. (6) is derived according to Storch et al. (2012) from the temperature $T$ and salinity $S$ transport and the equation of state $\rho=\rho(T, S, z)$. The variable $N$ in Eq. (6) is the buoyancy frequency defined by

$$
N^{2}=-\frac{g}{\rho_{0}} \frac{d \rho_{r}}{d z} .
$$

We now split the flow into time-varying and timemean components, denoted by primes and overbars, respectively. Throughout the paper the term "eddy" is used to refer to the time-varying components of the flow. The kinetic energy density is decomposed as (Chen et al. 2016; Kang and Curchitser 2017):

$$
E_{k}=\frac{1}{2} \underbrace{\rho_{0}\left(\bar{u}^{2}+\bar{v}^{2}\right)}_{E_{k 0}}+\frac{1}{2} \underbrace{\rho_{0}\left(u^{\prime 2}+v^{\prime 2}\right)}_{E_{k}^{\prime}}+\underbrace{\rho_{0}\left(\bar{u} u^{\prime}+\bar{v} v^{\prime}\right)}_{E_{k 0}^{\prime}},
$$

where mean kinetic energy (MKE) and the eddy kinetic energy (EKE) are defined as the time average of the $\overline{E_{k 0}}$ and $\overline{E_{k}^{\prime}}$ terms

$$
\begin{aligned}
& \mathrm{MKE}=\overline{E_{k 0}}=\frac{1}{2} \rho_{0}\left(\bar{u}^{2}+\overline{\boldsymbol{v}}^{2}\right), \\
& \mathrm{EKE}=\overline{E_{k}^{\prime}}=\frac{1}{2} \rho_{0} \overline{\left(u^{\prime 2}+v^{\prime 2}\right)},
\end{aligned}
$$

while the term $\overline{E_{k 0}^{\prime}}=0$ vanishes during time averaging.

Similarly also the available potential energy (APE; Gill 1982; Chen et al. 2016) is split into time-varying and time-mean components:

$$
\mathrm{APE}=\underbrace{\frac{g^{2} \bar{\rho}_{a}^{2}}{2 \rho_{0} N^{2}}}_{E_{p 0}}+\underbrace{\frac{g^{2} \rho_{a}^{\prime 2}}{2 \rho_{0} N^{2}}}_{E_{p}^{\prime}}+\underbrace{\frac{g^{2} \bar{\rho}_{a} \rho_{a}^{\prime}}{\rho_{0} N^{2}}}_{E_{p}^{\prime}} .
$$

The mean available potential energy (MPE) and eddy available potential energy (EPE) are defined as the time average of the $\overline{E_{p 0}}$ and $\overline{E_{p}^{\prime}}$ terms

$$
\begin{aligned}
& \mathrm{MPE}=\overline{E_{p 0}}=\frac{g^{2} \bar{\rho}_{a}^{2}}{2 \rho_{0} N^{2}}, \\
& \mathrm{EPE}=\overline{E_{p}^{\prime}}=\frac{g^{2} \overline{\rho_{a}^{\prime 2}}}{2 \rho_{0} N^{2}},
\end{aligned}
$$

while the term $\overline{E_{p 0}^{\prime}}=0$ vanishes during time averaging. MPE and EPE terms store energy in the tilted isopycnals (Chen et al. 2014). It can be easily proved the equality $\rho_{a}^{\prime}=\rho^{\prime}$, which means that $\rho_{a}^{\prime}$ is not dependent on the reference density and the choice of the domain (KC15).

The EKE budget equation with its sources, sinks and energy conversion terms is derived by multiplying Eqs. (1) and (2) by $\rho_{0} u^{\prime}$ and $\rho_{0} v^{\prime}$, respectively, and then taking the time average of their sum to give

$$
\begin{aligned}
\frac{\partial \mathrm{EKE}}{\partial t}= & \underbrace{-\nabla \cdot\left(\overline{\bar{u}^{\prime} p^{\prime}}\right)}_{\mathrm{PW}}-\nabla \cdot\left(\overline{\mathbf{u} E_{k}^{\prime}}\right)-\underbrace{-g \overline{\rho_{a}^{\prime} w^{\prime}}}_{\mathrm{EPE} \rightarrow \mathrm{EKE}} \\
& -\underbrace{\rho_{0}\left(\overline{u^{\prime} \mathbf{u}^{\prime}} \cdot \nabla \bar{u}+\overline{v^{\prime} \mathbf{u}^{\prime}} \cdot \nabla \bar{v}\right)}_{\mathrm{MKE} \rightarrow \mathrm{EKE}} \\
& +\underbrace{\rho_{0}\left(\overline{\mathbf{u}_{H}^{\prime} \cdot \widetilde{F}_{H}^{\prime}}+\overline{\mathbf{u}_{H}^{\prime} \cdot \mathscr{D}_{H}^{\prime}}\right)}_{F^{\prime} \& \mathrm{TD}^{\prime}} .
\end{aligned}
$$

The first terms on the left-hand side (LHS) of Eqs. (14)-(17), denote the tendency of EKE, MKE, EPE, and MPE, respectively. On the other hand, the last 
terms on the right-hand side (RHS; $F \&$ TD) of Eqs. (14)-(17), denote change rates of EKE, MKE, EPE, and MPE due to forcing, density sources, and turbulent diffusivity (TD) induced by the subgrid processes (Stepanov 2018). In Eq. (14), the first term on the RHS, $-\nabla \cdot\left(\overline{\mathbf{u}^{\prime} p^{\prime}}\right)$, represents the eddy pressure work $\left(\mathrm{PW}^{\prime}\right)$ and was split into its horizontal $-\nabla_{h}$. $\overline{\mathbf{u}_{h}^{\prime} p^{\prime}}$ and vertical $-\partial \overline{w^{\prime} p^{\prime}} / \partial z$ component as introduced by Zhai and Marshall (2013). Here, $\mathbf{u}_{h}$ are the horizontal velocities and $\nabla_{h}$ is the horizontal gradient operator. The second term, $-\nabla \cdot\left(\overline{\mathbf{u} E_{k}^{\prime}}\right)$, denotes the change of the EKE induced by the current advection and was also split into its horizontal $-\nabla_{h} \cdot \overline{\mathbf{u}_{h} E_{k}^{\prime}}$ and vertical $-\partial \overline{w E_{k}^{\prime}} / \partial z$ component. The third term, $-g \overline{\rho_{a}^{\prime} w^{\prime}}(\mathrm{EPE} \rightarrow \mathrm{EKE})$, denotes the rate of energy conversion from EPE to EKE and measures the strength of baroclinic instability (Stepanov 2018; Zhai and Marshall 2013; Zhan et al. 2016). The EPE is converted to EKE with denser fluid sinking or buoyant fluid rising. The fourth term, $\rho_{0}\left(\overline{u^{\prime} \mathbf{u}^{\prime}} \cdot \nabla \bar{u}+\overline{v^{\prime} \mathbf{u}^{\prime}} \cdot \nabla \bar{v}\right)($ MKE $\rightarrow$ EKE), represents the EKE change rate due to eddy momentum fluxes (Chen et al. 2014) by Reynolds stress (Orlanski and Cox 1972) associated with barotropic instabilities (KC15). The MKE budget equation is derived by multiplying Eqs. (1) and (2) by $\rho_{0} \bar{u}$ and $\rho_{0} \bar{v}$, respectively, and then taking the time average of their sum to give

$$
\begin{aligned}
\frac{\partial \mathrm{MKE}}{\partial t}= & \underbrace{-\nabla \cdot\left(\overline{\mathbf{u}} \overline{p_{a}}\right)}_{\mathrm{PW}}-\nabla \cdot(\overline{\mathbf{u M K E}}) \\
& \underbrace{-g \overline{\rho_{a}} \bar{w}}_{\mathrm{MPE} \rightarrow \mathrm{MKE}} \underbrace{-\rho_{0}\left[\bar{u} \nabla \cdot\left(\overline{\mathbf{u}^{\prime} u^{\prime}}\right)+\bar{v} \nabla \cdot\left(\overline{\mathbf{u}^{\prime} v^{\prime}}\right)\right]}_{\text {EKE } \rightarrow \mathrm{MKE}} \\
& +\underbrace{\underbrace{}_{0}\left(\overline{\mathbf{u}_{H}} \cdot \overline{\mathscr{T}} \bar{H}_{H}+\overline{\mathbf{u}_{H}} \cdot \overline{\mathscr{D}_{H}}\right)}_{\bar{F} \& \overline{\mathrm{TD}}} .
\end{aligned}
$$

In Eq. (15), the first term on the RHS, $-\nabla \cdot\left(\overline{\mathbf{u}} \overline{p_{a}}\right)$, represents the mean pressure work $(\overline{\mathrm{PW}})$ and was split in its horizontal $-\nabla_{h} \cdot\left(\overline{\mathbf{u}_{h}} \overline{p_{a}}\right)$ and vertical $-\partial \bar{w} \overline{p_{a}} / \partial z$ component. The second term, $-\nabla \cdot(\overline{\mathbf{u}} \mathrm{MKE})$, denotes the change of the MKE induced by the mean current advection and was split into its horizontal $-\nabla_{h} \cdot\left(\overline{\mathbf{u}_{h}} \mathrm{MKE}\right)$ and vertical $-\partial \bar{w} \mathrm{MKE} / \partial z$ component. The third term, $-g \overline{\rho_{a}} \bar{w}(\mathrm{MPE} \rightarrow \mathrm{MKE})$ denotes the rate of energy conversion from MPE to MKE through mean buoyancy flux (KC15). The fourth term, $-\rho_{0}\left[\overline{\mathbf{u}} \nabla \cdot\left(\overline{u^{\prime} u^{\prime}}\right)+\bar{v} \nabla \cdot\left(\overline{\mathbf{u}^{\prime} v^{\prime}}\right)\right]$ (EKE $\rightarrow$ MKE), represents the MKE change rate due to eddy momentum fluxes (Chen et al. 2014) via the work of Reynolds stress (KC15).

The EPE and MPE budget equations are derived by multiplying the density transport Eq. (6) by $g^{2} \rho_{a}^{\prime} / \rho_{0} N^{2}$ and $g^{2} \bar{\rho}_{a} / \rho_{0} N^{2}$, respectively, and then taking the time average to give

$$
\begin{aligned}
& \frac{\partial \mathrm{EPE}}{\partial t}=-\nabla \cdot\left(\overline{\mathbf{u} E_{p}^{\prime}}\right)+\underbrace{g \overline{\rho_{a}^{\prime} w^{\prime}}}_{\mathrm{EKE} \rightarrow \mathrm{EPE}}-\underbrace{\frac{g^{2}}{\rho_{0} N^{2}} \overline{\rho_{a}^{\prime} \mathbf{u}^{\prime}} \cdot \nabla \overline{\rho_{a}}}_{\mathrm{MPE} \rightarrow \mathrm{EPE}} \\
& +\underbrace{\frac{g^{2}}{\rho_{0} N^{2}}\left(\overline{\rho_{a}^{\prime} \mathscr{F}_{\rho}^{\prime}}+\overline{\rho_{a}^{\prime} \mathscr{D}_{\rho}^{\prime}}\right)}_{S^{\prime} \& \mathrm{TD}^{\prime}} \\
& \frac{\partial \mathrm{MPE}}{\partial t}=-\nabla \cdot(\overline{\mathbf{u M P E}})+\underbrace{g \overline{\rho_{a}} \bar{w}}_{\mathrm{MKE} \rightarrow \mathrm{MPE}}-\underbrace{\frac{g^{2}}{\rho_{0} N^{2}} \overline{\rho_{a}} \nabla \cdot\left(\overline{\mathbf{u}^{\prime} \rho_{a}^{\prime}}\right)}_{\mathrm{EPE} \rightarrow \mathrm{MPE}} \\
& +\underbrace{\frac{g^{2}}{\rho_{0} N^{2}}\left(\overline{\rho_{a}} \overline{\mathscr{F}_{\rho}}+\overline{\rho_{a}} \overline{\mathscr{D}_{\rho}}\right)}_{\bar{S} \& \overline{\mathrm{TD}}} .
\end{aligned}
$$

In Eq. (16), the first term in the RHS, $-\nabla \cdot\left(\overline{\mathbf{u} E_{p}^{\prime}}\right)$, represents the change of the EPE induced by the mean advection and was split in its horizontal $-\left(\nabla_{h} \cdot \overline{E_{p}^{\prime} \mathbf{u}_{h}}\right)$ and vertical $-\partial \overline{E_{p}^{\prime} w} / \partial z$ component, while the first term on the RHS in Eq. (17), $-\nabla \cdot(\overline{\mathbf{u}} \mathrm{MPE})$, represents the change of the MPE induced by the mean current advection and was also split in its horizontal $-\left[\nabla_{h} \cdot\left(\overline{\mathbf{u}_{h}} \mathrm{MPE}\right)\right]$ and vertical $-\partial \bar{w} \mathrm{MPE} / \partial z$ component. The second terms in Eqs. (16) and (17), $+g \overline{\rho_{a}^{\prime} w^{\prime}}(\mathrm{EKE} \rightarrow \mathrm{EPE})$ and (MPE $\rightarrow$ MKE) appear with opposite signs also in Eqs. (14) and (15), respectively. Hence these terms represent no net loss of kinetic or potential energy but a conversion from potential to kinetic energy consequently the increasing of the first means the decreasing of the second and a direct transfer of energy from one reservoir to the other. The third terms in Eqs. (16) and (17), $-g^{2} / \rho_{0} N^{2} \overline{\rho_{a}^{\prime} \mathbf{u}^{\prime}} \cdot \nabla \overline{\rho_{a}}(\mathrm{MPE} \rightarrow$ EPE) and $-g^{2} / \rho_{0} N^{2} \overline{\rho_{a}} \nabla \cdot\left(\overline{\mathbf{u}^{\prime} \rho_{a}^{\prime}}\right)(\mathrm{EPE} \rightarrow$ MPE$)$ are eddy mean flow interaction terms related to eddy density fluxes (Chen et al. 2014) and are associated with baroclinic instability (Zhan et al. 2016).

It is important to note that the terms MPE $\rightarrow$ EPE and $\mathrm{MKE} \rightarrow \mathrm{EKE}$ are not just negative values of the terms EPE $\rightarrow$ MPE and EKE $\rightarrow$ MKE, hence they do not represent a direct energy transport from one energy reservoir to the other. On the other hand, these conversion terms are related by a divergence form as

$$
\begin{gathered}
\int_{V}(\mathrm{EKE} \rightarrow \mathrm{MKE}+\mathrm{MKE} \rightarrow \mathrm{EKE}) d v \\
=-\int_{V} \nabla \cdot\left(\overline{\mathbf{u}^{\prime} E_{k 0}^{\prime}}\right) d v, \\
\int_{V}(\mathrm{EPE} \rightarrow \mathrm{MPE}+\mathrm{MPE} \rightarrow \mathrm{EPE}) d v \\
=-\int_{V} \nabla \cdot\left(\overline{\mathbf{u}^{\prime} E_{p 0}^{\prime}}\right) d v,
\end{gathered}
$$

where the integral sign represents the integration over an oceanic region. These relationships were studied by 
Harrison and Robinson (1978) and by Chen et al. (2014). Harrison and Robinson (1978) examined the transfer of energy between MKE and EKE reservoirs and reported that in an arbitrary volume of fluid, the changes in MKE and EKE are not generally equal and opposite. While Chen et al. (2014) stated that if the magnitudes of the RHS of Eq. (18) are negligible, then almost all the energy released from the mean flow is converted to eddy energy in the same region and the eddy-mean flow interaction is called local. This is automatically true in a closed ocean region. On the other hand, if their magnitudes are not negligible, part of the energy released from the mean flow that is not used to sustain the local eddy energy growth is transported outside of the domain.

\section{b. Model configuration and validation}

The ocean model used in this study is the Regional Ocean Modeling System (ROMS, Shchepetkin and McWilliams 2005). ROMS is a three-dimensional, terrain-following, free-surface, ocean circulation model, which uses hydrostatic and Boussinesq approximation to solve the Reynolds-averaged Navier-Stokes equations. The model domain extends from $97.7^{\circ}$ to $79^{\circ} \mathrm{W}$ and from $15.6^{\circ}$ to $30.5^{\circ} \mathrm{N}$. The model is implemented with a horizontal resolution of about $5 \mathrm{~km}$ and 36 vertical terrainfollowing levels. Vertical eddy diffusivity was computed using the Mellor-Yamada 2.5 scheme (Mellor and Yamada 1982; Galperin et al. 1988), while the horizontal diffusion was represented using a Smagorinsky (1963) eddy parameterization. A free-running simulation was integrated for the calendar years of 2010-14 (5 years total), and its daily mean results are analyzed in this study. This model has been used previously to study the connectivity of the deep GoM (Maslo et al. 2020), where the model was validated for the deep Gulf using 158 floats drifting between 1500- and 2500-m depth and current velocity data from 28 moorings. We encourage the reader to turn to this publication for more information about the simulation configuration.

As discussed in the introduction, the dynamics of the upper layer affect the energetics of the deep layer. Therefore, a validation of the model in the upper layer is also recommended. In this regard, the surface MKE and EKE obtained from the geostrophic currents provided by Archiving, Validation and Interpretation of Satellite Oceanographic data (AVISO; https://www.aviso.altimetry.fr/ en/my-aviso.html), and the geostrophic currents calculated from ROMS altimetry are compared to determine if they present a similar distribution and overall magnitude of values to be considered suitable for use in the energetics analysis. The AVISO dataset has a spatial resolution of $0.25^{\circ}$ and a temporal resolution of 1 day (January 2010-June 2014), while the spatial resolution of the ROMS altimetry has been reduced to match the one from AVISO.

Comparing the mean LC position, denoted by the 17-cm sea surface high (SSH) contour (Leben 2013) of the model and AVISO results (Figs. 1a,b), one can see that the model results match reasonably well the elongation and thickness of the mean LC position obtained from the observations. Both the model and observed MKE distribution presents its highest values in the area of the LC where observed maximum values reach $500 \mathrm{~J} \mathrm{~m}^{-3}$ (Fig. 1b) and the modeled $600 \mathrm{~J} \mathrm{~m}^{-3}$ (Fig. 1a). Another point to observe is that the MKE derived from observation is predominantly concentrated in the eastern part of the Gulf while the model displays an additional current close to the west coast with MKE values reaching $150 \mathrm{~J} \mathrm{~m}^{-3}$. Similarly the EKE obtained from the satellite observation (Fig. 1d) is concentrated in the eastern part of the GoM (eastward of $90^{\circ} \mathrm{W}$ ) with its values reaching $300 \mathrm{~J} \mathrm{~m}^{-3}$ while the model EKE (Fig. 1c) displays lower energy values $\left(\sim 200 \mathrm{~J} \mathrm{~m}^{-3}\right)$ and its distribution extend deep into the western part of the Gulf. Although there are differences in distribution and range between the model and the observed MKE and EKE, it can be assumed that the simulated circulation in GoM is sufficiently accurate and is therefore considered appropriate for the following energetics analysis.

\section{Results}

\section{a. Energy cycle}

Equations (14)-(17) include eight conversion terms that exchange energy among the energy reservoirs. These exchanges describe a cycle of energy, which is often represented in a schematic diagram called the Lorenz energy cycle (Lorenz 1955). A traditional Lorenz energy cycle is very often used to illustrate the energy pathways in the global oceans and atmosphere or in a closed region where the divergence terms in Eq. (18) vanish resulting in a direct conversion of energy and a simpler energy diagram. However, in the open regions it is also necessary to take into consideration the transport of energy due to advection and due to the divergence of the pressure work. The latter adds some complexity to the diagram and changes its simple shape. Accordingly, we prefer to omit the term Lorenz energy cycle to avoid confusion.

It is important to emphasize that the values of the reference density $\rho_{r}$ and reference pressure $p_{r}$ in the energy equations depend on the choice of the area to be analyzed. Thus, in this work, we analyzed only the area of the Gulf bounded by the Yucatan and Florida straits, as denoted by the dashed red lines in Fig. 1. 
(a)

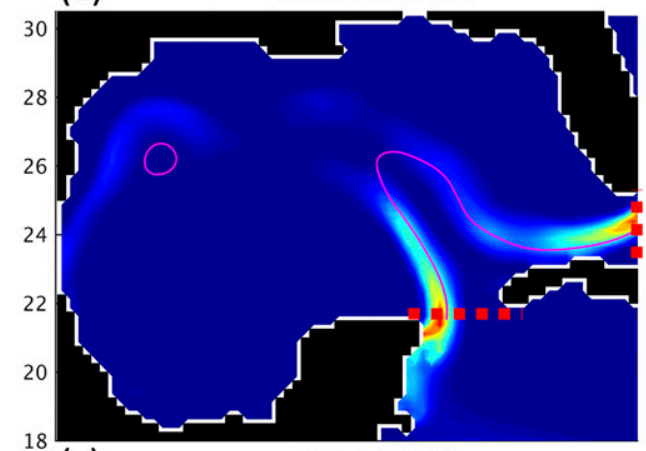

(c)

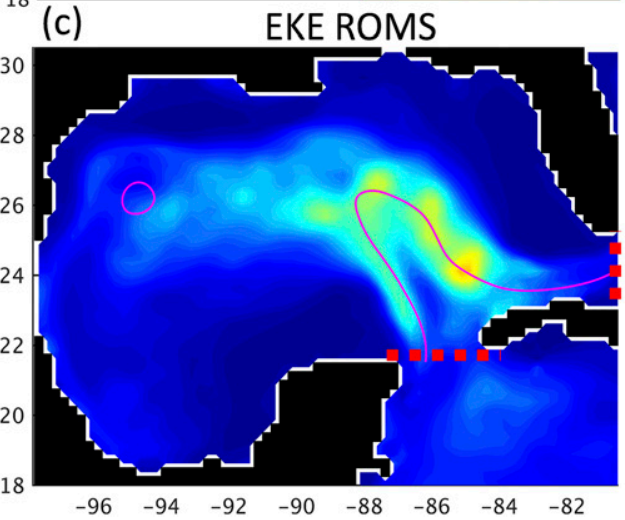

(b)

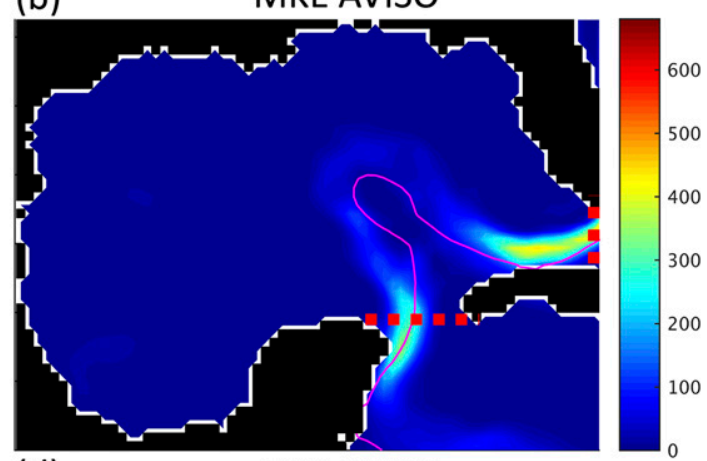

(d)

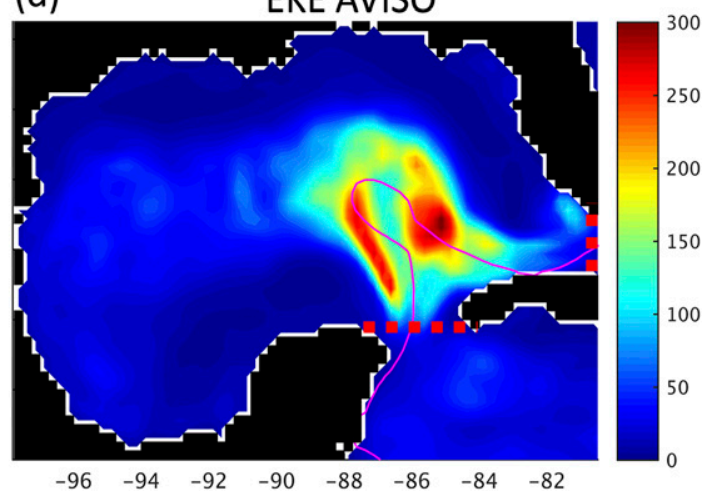

FIG. 1. Horizontal distribution of energy densities $\left(\mathrm{J} \mathrm{m}^{-3}\right)$ computed from the surface geostrophic currents obtained from (a),(c) ROMS altimetry and (b),(d) AVISO dataset. The spatial resolution of ROMS altimetry data has been reduced to match the resolution of the AVISO dataset. The purple line shows the mean boundary (17-cm SSH contour) of the LC for the selected dataset. Dashed red lines denote the area of the Gulf analyzed in this study.

Since ROMS uses a generalized vertical terrain-following coordinate system, the velocities were linearly interpolated to 33 depths. These depths mimic the standard oceanic depths (Levitus 1982) except on the first layer where we chose to start at a depth of $1 \mathrm{~m}$ to avoid blank values due to free surface fluctuation. Using the interpolated model output, we evaluated the four ocean energy components MKE, EKE [Eqs. (9) and (10)], MPE, and EPE [Eqs. (12) and (13)] as well the energy conversion terms Eqs. (14)-(17). It is important to note that the last terms on the RHS of Eqs. (14)-(17) representing the change rates of energy due to forcing, density sources and turbulent diffusivity are not explicitly evaluated from the model results but evaluated from Eqs. (14)(17) based on the fact that all other terms have been evaluated.

The area-mean energy densities of energy reservoirs are displayed in Fig. 2. As mentioned in the introduction, the depth of $1000 \mathrm{~m}$ is usually adopted to divide the Gulf into two layers. It is interesting to note that also from an energy analysis perspective, the depth of $1000 \mathrm{~m}$ represents a clear distinction between the upper and the deeper layer. In the upper layer, all reservoir energy densities are of the same order of magnitude decreasing exponentially until the 1000-m depth. Under the 1000-m depth EKE and MKE (KE) remains approximately constant, while MPE and EPE (PE) continue to decrease until the

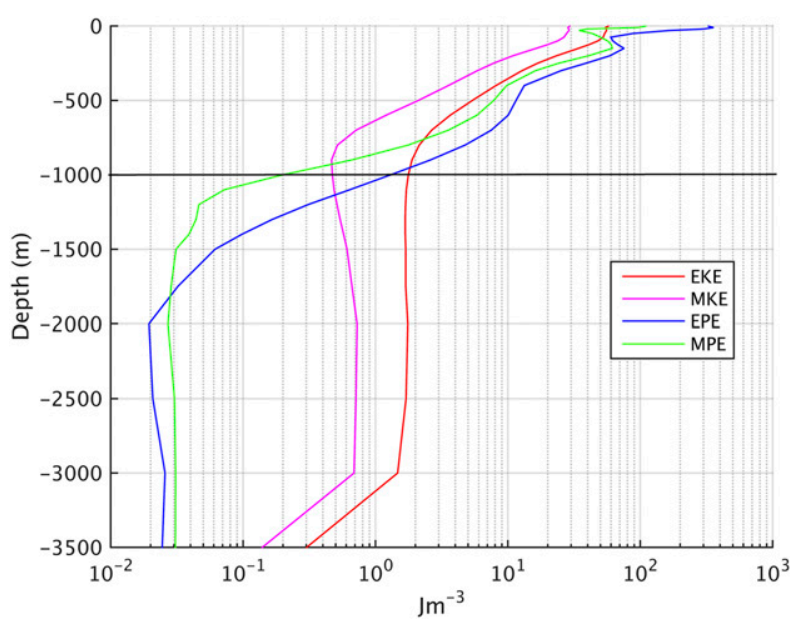

FIG. 2. Vertical distribution of the area-mean energy densities averaged over the GoM. 

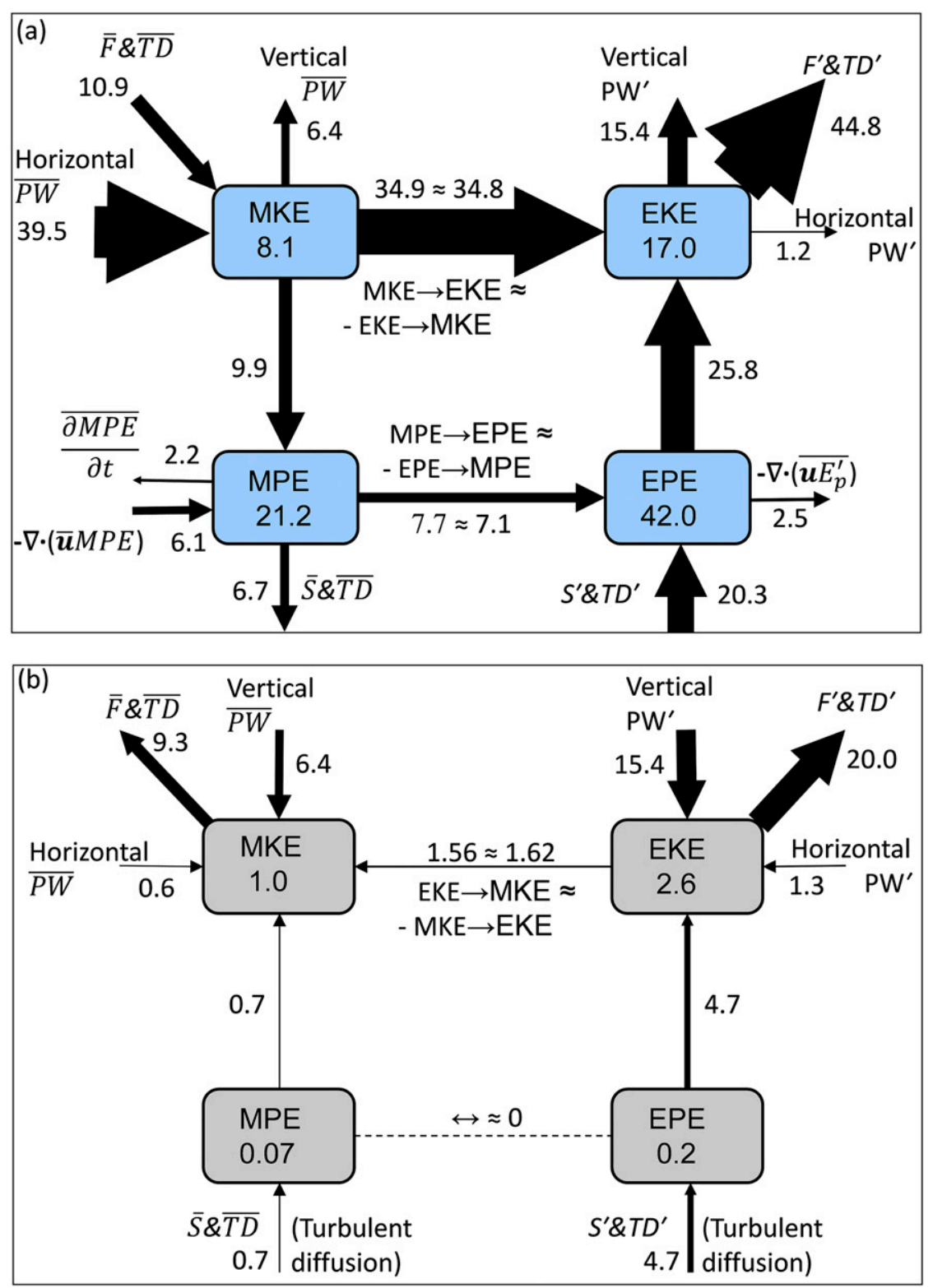

FIG. 3. Schematic of the energy diagram (volume integration) over the (a) upper layer and (b) deep GoM layer. Energy reservoirs are given in units of petajoules $\left(\mathrm{PJ}=10^{15} \mathrm{~J}\right)$ and the rates of generation, dissipation, and conversion [Eqs. (14)-(17)] are in $10^{8} \mathrm{~W}$.

1500- and 2000-m depths, respectively. This means that under $1000 \mathrm{~m}$ the $\mathrm{PE}$ terms receive less energy inflow to sustain themselves in respect to $\mathrm{KE}$ reservoirs. Very similar results were found by $\mathrm{KC} 15$ in their Gulf Stream along-coast region (see Fig. 5c in $\mathrm{KC} 15$ ).

The energy transfer diagram in Fig. 3 displays only the dominant (nonnegligible) terms from Eqs. (14)-(17) separately for the upper and the deeper layer of the Gulf, while the energy diagram for the whole Gulf can be obtained by summing the two parts together. These terms have at least an order of magnitude higher values than the neglected terms.

The dominant energy source for the upper layer is the MKE horizontal $\overline{\mathrm{PW}}\left(39.5 \times 10^{8} \mathrm{~W}\right)$. The $\mathrm{PW}$ has a divergence form and is zero in a closed domain, which means that the energy is entering into the Gulf with the LC. Another source of energy in the Gulf is the wind, which has not been explicitly evaluated in this work but one can observe its effect in the $\bar{F} \& \overline{\mathrm{TD}}$ term, where the latter displays a positive value $\left(10.9 \times 10^{8} \mathrm{~W}\right)$ because the generation of energy due to wind is higher than the 
dissipation of energy due to turbulence. Because the LC spatial variability at the Yucatan and Florida straits is constrained by the coast, the EKE horizontal $\mathrm{PW}^{\prime}$ contribution to the EKE generation in the Gulf is minor.

In the upper layer, the mean flow drives the eddy flow, where the EKE $\rightarrow$ MKE conversion term releases the energy from the mean flow and the MKE $\rightarrow$ EKE term is used to convert this energy into the eddies. The whole energy transfer related to barotropic instabilities $(\mathrm{MKE} \rightarrow \mathrm{EKE})$ in the upper layer amounts to $34.9 \times$ $10^{8} \mathrm{~W}$ while the sum of energy transfer related to baroclinic instabilities (EPE $\rightarrow$ EKE) amounts to $25.9 \times$ $10^{8} \mathrm{~W}$, which means that in the upper-layer barotropic instabilities or horizontal shear is the dominant generator of eddies. Typically, the eddy generation from MKE is believed to be of importance for regions characterized by an intense current of about $1 \mathrm{~m} \mathrm{~s}^{-1}$ as seen in the Labrador Sea (Eden and Böning 2002), the Gulf Stream region (KC15) or South China Sea (Yang et al. 2013), while the baroclinic instability appears to be the most important mechanism in generating mesoscale eddies activity in most other parts of the ocean (Beckmann et al. 1994; Storch et al. 2012). Another thing to note is that the conversion term MKE $\rightarrow$ EKE is approximately equal to the term EKE $\rightarrow \mathrm{MKE}$ and $\mathrm{MPE} \rightarrow \mathrm{EPE}$ is approximately equal to EPE $\rightarrow$ MPE indicating that the Gulf is behaving like a closed domain and displaying a local conversion of energy.

An important part of analyzing the upper layer was to determine how the transfer of energy into the deep layer occurs. The energy is transported downward by vertical $\mathrm{PW}$ ( $\overline{\mathrm{PW}}$ and $\left.\mathrm{PW}^{\prime}\right)$, where the transport downward due to advection of EKE and MKE can be neglected. Because the Gulf in the vertical direction represents a closed region, with a free-surface on the upper side and a seafloor on the lower side, it can be shown using the divergence theorem that the sum of the vertical PW in each water column is approximately equal to zero and a divergence of vertical PW in the upper layer must result in a convergence of vertical PW energy in the lower layer and vice versa. The evidence for this statement is presented in the appendix.

About $75 \%$ of the energy sustaining the deep KE is transferred from the upper to the deep layer by the vertical PW, approximately $6 \%$ by the horizontal $\mathrm{PW}$ through the Yucatan and Florida straits and $\sim 19 \%$ is generated through the processes related to baroclinic instabilities. The vertical $\mathrm{PW}^{\prime}$ generated by the eddy flow is more than 2 times higher than the vertical $\overline{\mathrm{PW}}$ generated by the mean flow, meaning that the eddies have a higher effect on the energy flux into the deep layer. It is interesting to note that in the upper layer all the energy reservoirs are of the same order of magnitude, while in the deep layer APE terms (EPE and MPE) are an order of magnitude smaller than the KE reservoirs, and that unlike in the case of KE terms, the amount of vertical energy influx to the deep APE terms is negligible. The lower magnitude of APE terms in the deep layer is somewhat expected because the APE vanishes in the horizontal and statistically stable stratification (Lorenz 1955), and one can expect that the deep layer has a more horizontal and stable stratification than the upper layer because of its position mostly below the LC. The APE in the deep layer is generated predominately by the turbulent diffusion term ( $S \&$ TD), where the subgrid diffusion (parameterized in the model) is destabilizing the stratification by diffusing the water from a denser layer into a lighter one and consequently changing the perturbation density $\rho_{a}$ in Eqs. (12) and (13), resulting in an increase of APE in the deep layer. It is important to point out that density sources $\left[\mathscr{S}_{\rho}\right.$ in Eq. (6)] were not included in the deep layer and that the contribution from the $S \&$ TD terms is purely from the turbulent diffusion.

In the deep layer, the eddy flow drives the mean flow, where the MKE $\rightarrow$ EKE barotropic term releases the energy from the eddies, and the EKE $\rightarrow$ MKE term is used to convert this energy into the mean flow. This is the opposite of the mechanism found in the upper layer. Although this process is important in sustaining the MKE reservoir its contribution of about $1.6 \times 10^{8} \mathrm{~W}$ is much smaller than the contribution of the vertical PW of approximately $6.4 \times 10^{8} \mathrm{~W}$. Another difference with the upper layer is that in the deep layer the baroclinic conversion term $\mathrm{EPE} \rightarrow \operatorname{EKE}\left(4.7 \times 10^{8} \mathrm{~W}\right)$ is higher than the barotropic conversion term EKE $\rightarrow$ MKE $\left(1.6 \times 10^{8} \mathrm{~W}\right)$ and that the energy conversion due to vertical shear is higher than the conversion due to horizontal shear. The conversion terms MPE $\rightarrow$ EPE and EPE $\rightarrow$ MPE in the deep layer are negligible while the term MKE $\rightarrow$ EKE is approximately equal to the term EKE $\rightarrow$ MKE indicating a local conversion of energy also in the deep layer. In the end, the energy of the deep GoM is dissipated through the $\bar{F} \& \overline{\mathrm{TD}}$ and $F^{\prime} \& \mathrm{TD}^{\prime}$ terms by the bottom drag and turbulent viscous stresses. Regarding the analysis of the energy cycle, we would also like to point out that the MPE $\rightarrow$ EPE and EPE $\rightarrow$ EKE conversion terms (in the upper and lower layer) are not in the same order of magnitude (Fig. 3). The latter is not in line with a traditional baroclinic instability energy pathway (Youngs et al. 2017), usually present in the global-scale Lorenz energy cycles (Olbers et al. 2012).

\section{b. Energy generation and dissipation}

The goal of this section is to understand how the energy that drives the deep GoM circulation is maintained. 
Equations (14)-(17) describe energy inflow and outflow into each energy reservoir separately. The terms in this equation displaying positive values represent the energy flux into the reservoir and as such are sustaining the energy, while the terms with negative values represent the energy losses.

It is common in the literature to analyze only the EKE reservoir sources and sinks and to display the spatial distribution of each term in Eq. (14) in separate figures, usually resulting in six figures (Yang et al. 2013). Because we are examining all four energy reservoirs in two layers with a split horizontal and vertical transfer of energy, a complete analysis would amount to an overwhelming $(\sim 50)$ number of figures. The shortcoming of this kind of analysis is that it is difficult to summarize the results and understand which process is dominant in a specific part of the domain. To avoid this scenario an innovative approach has been adopted. Instead of examining each term in a separate figure and determine where each term is a source or sink of an energy reservoir based on its sign and strength, we decided to display all the terms corresponding to a specific energy reservoir in only two figures. The first figure focusing on the energy generation (energy sources) and the second figure on energy dissipation (energy sinks). To create the energy generation figure we searched in each computational cell of the Gulf for the term with the largest positive value, while in the energy dissipation figure we searched for the most negative term.

Because it is difficult to distinguish between more than four colors in a single figure, the mean horizontal $\overline{\mathrm{PW}}$ and the $\bar{F} \& \overline{\mathrm{TD}}$ term and the eddy horizontal $\mathrm{PW}^{\prime}$ and $S^{\prime} \& \mathrm{TD}^{\prime}$ term have been joined together. The reasons for this are twofold. The first reason is that this work focuses on understanding how the energy in the deep GoM is maintained and less on its dissipation. The second reason is that a similar merging has already been done in the work of Zhai and Marshall (2013), where they join together all the terms in Eq. (14) except the vertical $\mathrm{PW}^{\prime}$ and EKE source term EPE $\rightarrow$ EKE. The difference between our approach and that of Zhai and Marshall (2013), is that we also examined separately the barotropic MKE $\rightarrow$ EKE term and we did not join this term together with the other horizontal eddy energy fluxes.

\section{1) EKE GENERATION AND DISSIPATION}

The magnitude and the distribution of the EKE energy reservoir and the EKE conversion terms depth-integrated separately for the upper and lower layer is presented in Fig. 4. The upper-layer EKE reservoir (Fig. 4a) shows the highest values in the area of the LC and then gradually decreases its energy level toward the western Gulf, while maintaining higher values between the $25^{\circ}$ and $28^{\circ} \mathrm{N}$, where approximately $80 \%$ of LCEs go through (Vukovich 2007). Comparing the EKE distribution of the upper (Fig. 4a) and deeper layer (Fig. 4b) it can be observed that they share a similar form, possibly indicating the connectivity between the upper and deeper layer EKE. The deep EKE also displays a very similar distribution as the EKE produced by the drifting floats (see Figs. 3-38 in Hamilton et al. 2016). Higher values of EKE (Fig. 4a) occupy a larger area in the eastern-central part of the Gulf, while the higher MKE values (Fig. 5a) are mostly confined to the sharp boundary currents. A strong mean-to-eddy MKE $\rightarrow$ EKE conversion term (corn color in Fig. 4c) related to barotropic instabilities can be observed in the area of the LC from the Yucatan channel up to its tip. Similar results were also simulated by Garcia-Jove et al. (2016). Another noticeable color in Fig. $4 \mathrm{c}$ is red (EPE $\rightarrow$ EKE), denoting the EKE generation by the release of APE stored in the tilted isopycnals. The EPE $\rightarrow$ EKE term displays the highest values on the eastern half of the LC from its tip down to $24^{\circ} \mathrm{N}$ and in contrast to the MKE $\rightarrow$ EKE term, which is dominant on its western side. This term, although weaker, also seems to be important in the central part of the Gulf. As mentioned in the section 3a, the divergence of vertical $\mathrm{PW}^{\prime}$ (Fig. 4e) in the upper layer is directly connected to the convergence of the vertical $\mathrm{PW}^{\prime}$ in the deep layer (Fig. $4 \mathrm{~d}$ ), where a prevailing green color can be observed across the Gulf and especially inside the contour representing the mean LC position (Fig. 4d).

The latter confirms the quantitative results presented in section 3a that the vertical PW contributes to about $75 \%$ of the energy influx into the deep GoM. Also noticeable is the contribution of the horizontal $\mathrm{PW}^{\prime}+F^{\prime} \& \mathrm{TD}^{\prime}$ term. Nevertheless, the latter is mostly redistributing the energy with the horizontal $\mathrm{PW}^{\prime}$ across the deep Gulf as the total contribution of the $F^{\prime} \& \mathrm{TD}^{\prime}$ term is negative (Fig. 3b). The last term that has some influence in the generation of EKE in the deep layer is the EPE $\rightarrow$ EKE term related to baroclinic instabilities. The EPE $\rightarrow$ EKE term is most effective in disturbing the stable stratification under the area of the LC and over the area of the steep slopes along the $26^{\circ} \mathrm{N}$. These steep slopes also represent the locations where the deep eddies collide with the continental slope and release the energy (MKE $\rightarrow$ EKE in Fig. 4f) to generate MKE. Comparing Fig. $4 \mathrm{~d}$ and Fig. $4 \mathrm{f}$ it is possible to note that the areas of the generation of EKE due to vertical $\mathrm{PW}^{\prime}$ (Fig. 4d) are balanced by the dissipation of the energy by the horizontal $\mathrm{PW}^{\prime}+F^{\prime} \& \mathrm{TD}^{\prime}$ term (Fig. 4f). The latter indicates that the horizontal 
(a)
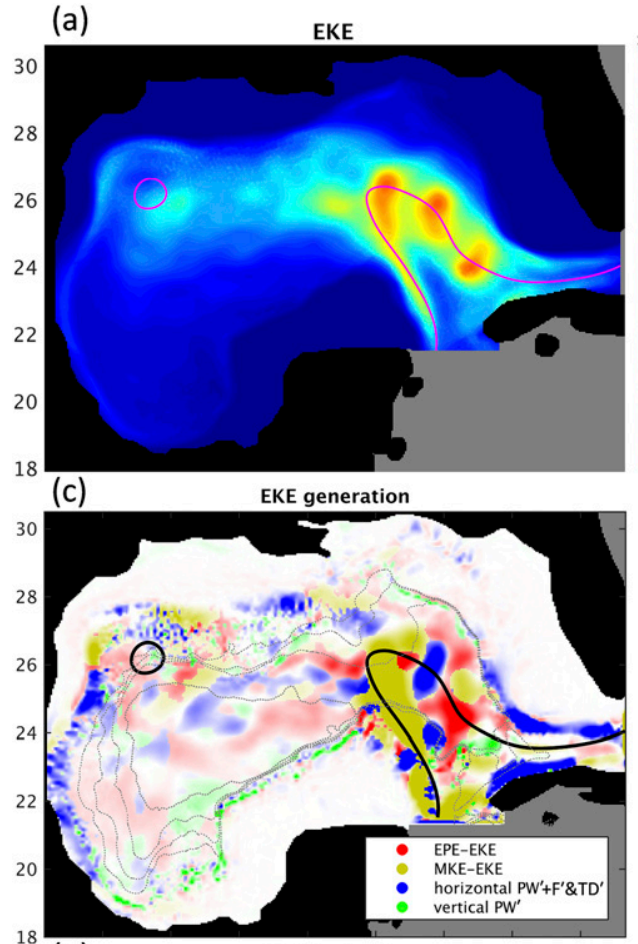

(e) (b)

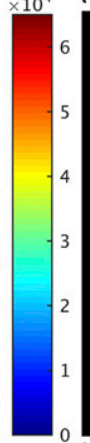

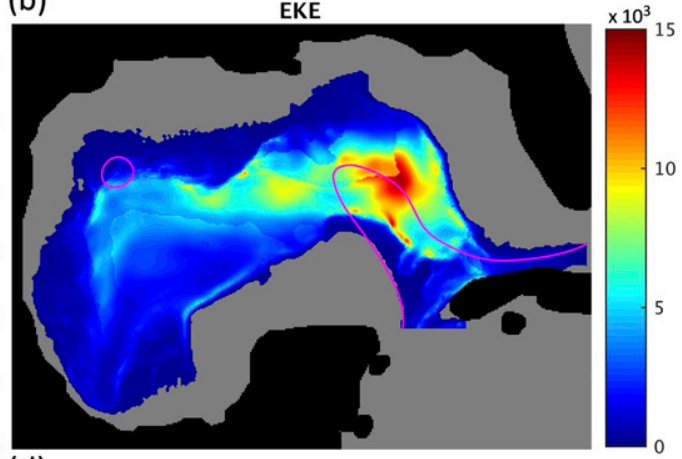

(d)

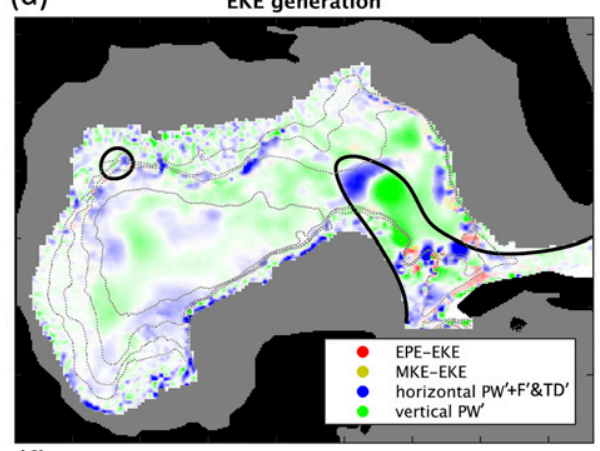

(f)

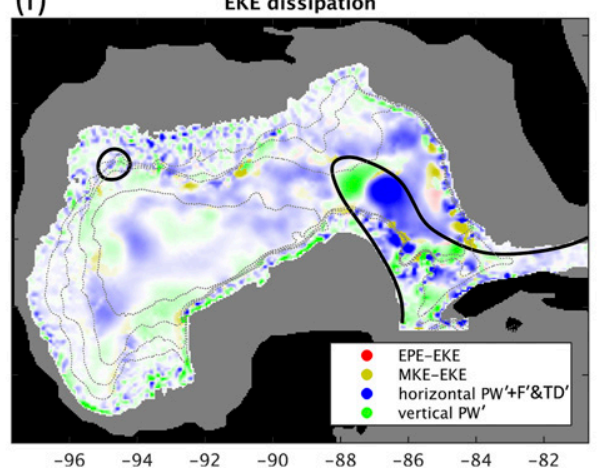

FIG. 4. Comparison of the horizontal distributions of the depth-integrated EKE reservoirs $\left(\mathrm{J} \mathrm{m}^{-2}\right)$ and the dominant EKE generation, dissipation, and conversion terms for the (left) upper layer and (right) deep layer. The most intense EKE generation colors represent values higher or equal to $0.04 \mathrm{~W} \mathrm{~m}^{-2}$, while the most intense EKE dissipation colors represent values smaller or equal to $-0.04 \mathrm{~W} \mathrm{~m}^{-2}$. The purple and black lines show the mean boundary (17-cm SSH contour) of the LC, and the bathymetry contours are spaced at $-2000,-2500,-3000$, and $-3500 \mathrm{~m}$.

$\mathrm{PW}^{\prime}+F^{\prime} \& \mathrm{TD}^{\prime}$ term redistribute and diffuse the energy inflow into the deep layer due to the vertical $\mathrm{PW}^{\prime}$.

\section{2) MKE GENERATION AND DISSIPATION}

The upper and lower EKE reservoirs (Figs. 4a,b) have a very similar distribution of energy, while the MKE reservoirs displays a very different distribution of energy between the upper and lower layer (Figs. 5a,b). In the upper layer, the strongest MKE values are following the shape of the mean boundary of the LC (Fig. 5a) while in the deep layer the MKE is concentrated in the areas of the sharp boundary currents (Fig. 5b). The latter is possibly indicating weaker energy connectivity between the upper- and lower-layer MKE energy reservoirs in comparison to the EKE reservoirs.

An important part of the energy inflow into the upper layer is redistributed and diffused across the Gulf by the horizontal $\overline{\mathrm{PW}}+\bar{F} \& \overline{\mathrm{TD}}$ term (Fig. 5c). The eddies tend to accelerate the mean flow (EKE $\rightarrow$ MKE) along the steep slopes in the northern part (west of $88^{\circ} \mathrm{W}$ ) of the Gulf and in the area close to the Florida strait (Fig. 5c), where the bathymetry constrains the spatial variability 
(a)
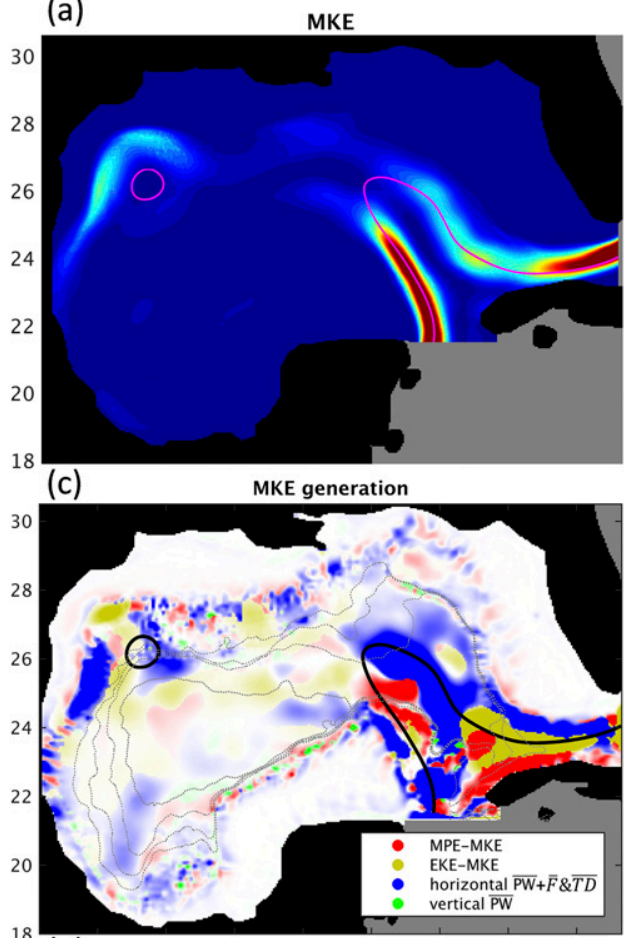

(e) MKE dissipation

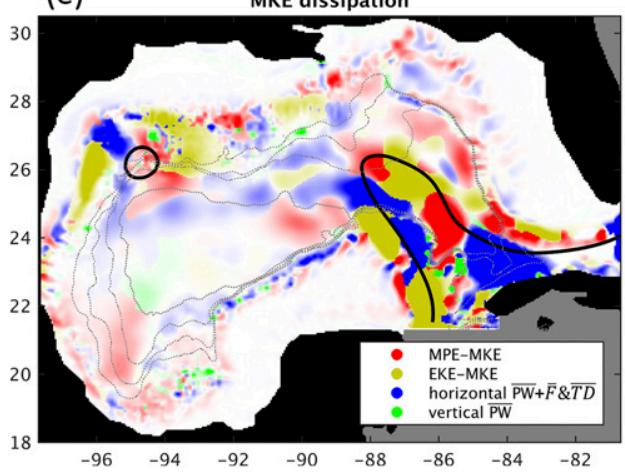

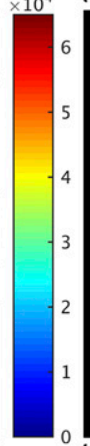

(b) MKE

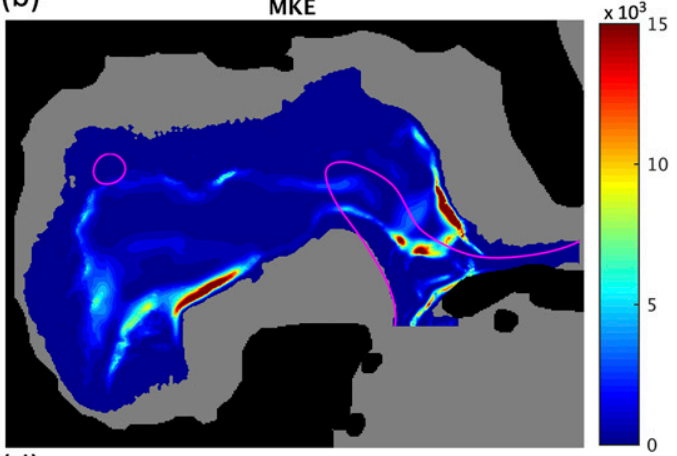

(d)

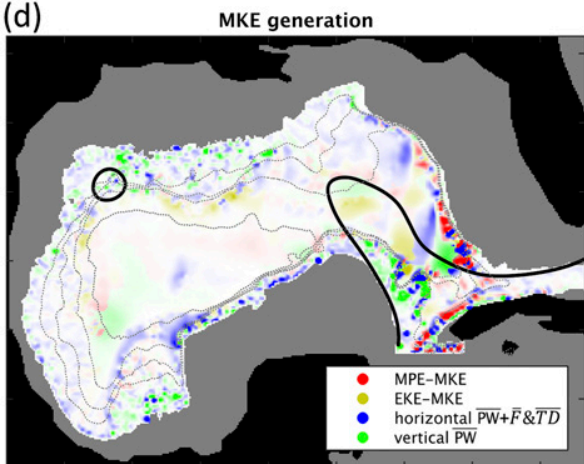

(f)

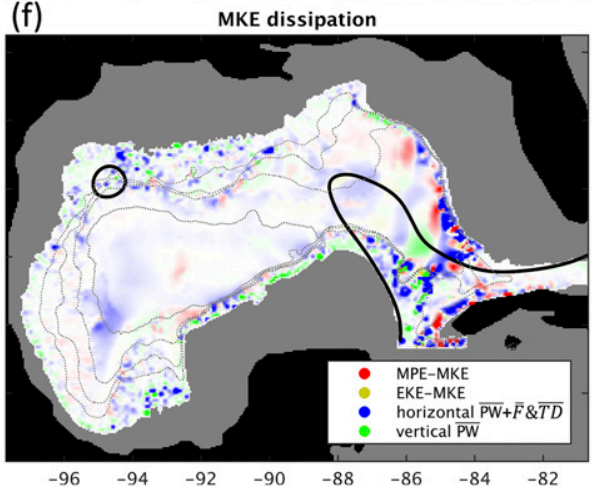

FIG. 5. Comparison of the horizontal distributions of the depth-integrated MKE reservoirs $\left(\mathrm{J} \mathrm{m}^{-2}\right)$ and the dominant MKE generation, dissipation, and conversion terms for the (left) upper layer and (right) deep layer. The most intense MKE generation colors represent values higher or equal to $0.03 \mathrm{~W} \mathrm{~m}^{-2}$, while the most intense MKE dissipation colors represent values smaller or equal to $-0.03 \mathrm{~W} \mathrm{~m}^{-2}$. The purple and black lines show the mean boundary (17-cm SSH contour) of the LC, and the bathymetry contours are spaced at $-2000,-2500,-3000$, and $-3500 \mathrm{~m}$.

of the flow. It is interesting to note an MPE $\rightarrow$ MKE area, close to the tip of the LC (Fig. 5c) denoting the generation of MKE from the energy stored in the mean stratification released by the horizontal $\overline{\mathrm{PW}}+\bar{F} \& \overline{\mathrm{TD}}$ term (Fig. 5e). The amount of mean vertical $\overline{\mathrm{PW}}$ transferred from the upper to the lower layer is more than two times smaller in comparison to the eddy vertical $\mathrm{PW}^{\prime}$ (Fig. 3). The latter is also reflected in Fig. 5e, where it is difficult to detect the divergence of vertical $\overline{\mathrm{PW}}$ due to its small amount. Nevertheless, minor quantities of vertical $\overline{\mathrm{PW}}$ can be observed in the regions of the steep slopes. Figure $5 \mathrm{e}$ also shows the area where the MKE is released to generate eddies (EKE $\rightarrow$ MKE), most noticeably in the area of the LC.

The EKE generation and dissipation terms of the deep layer (Figs. 4d,f) are roughly evenly distributed in the Gulf interior as well as on its boundary, while the MKE generation and dissipation terms (Figs. 5d,f) are mostly concentrated on its boundary, where the strongest boundary currents are located. The highest values of the mean vertical $\overline{\mathrm{PW}}$ can be observed in the areas of the rough bathymetry and in the areas of the steep slopes 
under the LC. As in the upper layer, the eddies act to accelerate the mean flow (EKE $\rightarrow$ MKE) along the steep slopes on the northern part (west of $88^{\circ} \mathrm{W}$ ) of the Gulf and in the area under the LC (Fig. 5d). About $0.7 \times 10^{8} \mathrm{~W}$ of MKE energy is generated in the deep also by MPE $\rightarrow$ MKE term extracting energy stored in the mean stratification mostly in the eastern Gulf. Another term that has an important role in maintaining the circulation in the deep Gulf is the horizontal $\overline{\mathrm{PW}}+\bar{F} \& \overline{\mathrm{TD}}$ term. The latter is sustaining the energy by its redistribution and diffusion and shows its highest values in the areas of the strongest southern and eastern boundary currents. Most of the energy is dissipated in the areas of rough bathymetry and the steep slopes (horizontal $\overline{\mathrm{PW}}+\bar{F} \& \overline{\mathrm{TD}}$ in Fig. 5f) especially in the western part of the Gulf. It can be seen in Fig. 5f, that some amount of MKE energy is converted back to MPE and stored in its mean stratification.

\section{3) EPE AND MPE GENERATION AND DISSIPATION}

Because in the cases of the EPE and MPE reservoirs the amount of energy transfer from the upper to the lower layer is negligible (Fig. 3), the upper-layer EPE and MPE terms are not relevant to this work and consequently will not be analyzed.

In comparison to the analysis of the EKE and MKE reservoirs where several terms are affecting their energy generation and dissipation, the EPE and MPE analysis is rather simple because there are only two terms affecting each energy reservoir. It can be seen in Fig. 6a that the highest values of EPE are located under the LC and in the northwestern corner of the Gulf, also called the "eddy graveyard" (Biggs et al. 1996). A noticeable area of higher EPE can also be observed in the western part of the Gulf in the area of the deep gyre (Maslo et al. 2020). It can be observed in Fig. $6 \mathrm{~b}$ that the EPE is generated mostly by the turbulent diffusion term $\left(S^{\prime} \& \mathrm{TD}^{\prime}\right)$ and by a lesser extent from the EKE $\rightarrow$ EPE term. The turbulent diffusion term is generating EPE in the areas of rough bathymetry, steep slopes, and under the LC. The only two nonnegligible terms in Eq. (16) in the deep GoM are the $S^{\prime} \& \mathrm{TD}^{\prime}$ and the EKE $\rightarrow$ EPE terms. This means that the $S^{\prime} \& \mathrm{TD}^{\prime}$ term is approximately equal to $-\mathrm{EKE} \rightarrow \mathrm{EPE}$, which itself is equal to EPE $\rightarrow$ EKE term in Eq. (14). The latter indicates that the EPE generated by the turbulent diffusion $\left(S^{\prime} \& \mathrm{TD}^{\prime}\right)$ is converted to EKE by the EPE $\rightarrow$ EKE term and is related to baroclinic instabilities. The distribution of EKE $\rightarrow$ EPE along the boundary presents both positive (Fig. 6c) and negative values (Fig. 6d), indicating the energy flux from the eddies to the mean stratification (Yang et al. 2013), which is related to the interaction between the flow and the topography.
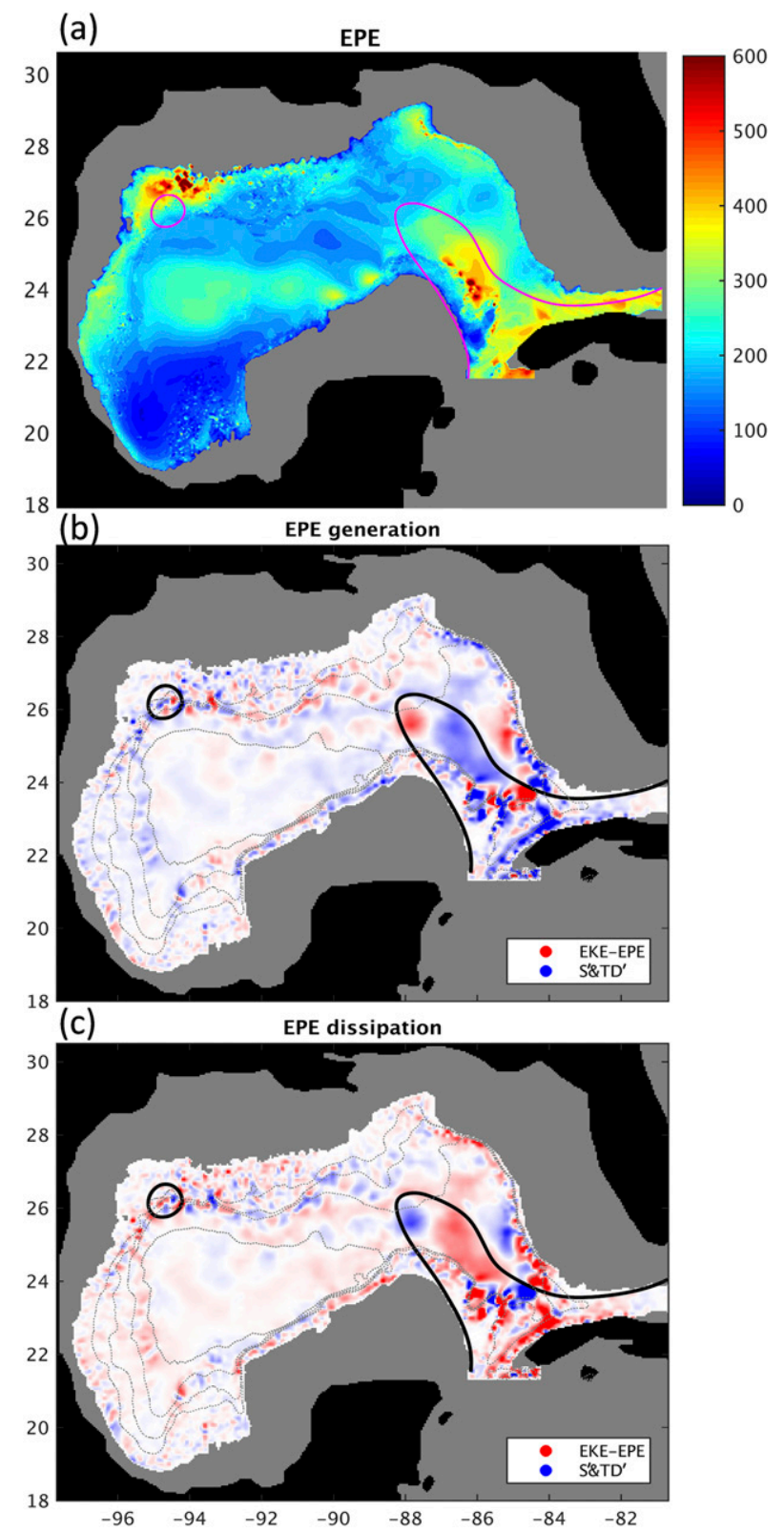

FIG. 6. Comparison of the horizontal distributions of the deep layer depth-integrated EPE reservoir $\left(\mathrm{J} \mathrm{m}^{-2}\right)$ and the dominant EPE generation, dissipation, and conversion terms. The most intense EPE generation colors represent values higher or equal to $0.01 \mathrm{~W} \mathrm{~m}^{-2}$, while the most intense EPE dissipation colors represent values smaller or equal to $-0.01 \mathrm{~W} \mathrm{~m}^{-2}$. The purple and black lines show the mean boundary (17-cm SSH contour) of the LC, and the bathymetry contours are spaced at $-2000,-2500$, -3000 , and $-3500 \mathrm{~m}$.

By comparing Figs. $6 \mathrm{~b}$ and $6 \mathrm{c}$ with Figs. $7 \mathrm{~b}$ and $7 \mathrm{c}$ a very similar color pattern can be observed indicating a similar energy conversion for the MPE reservoir. The turbulent diffusion term $(\bar{S} \& \overline{\mathrm{TD}})$ is generating MPE in the areas of rough bathymetry and steep slopes. 
(a)

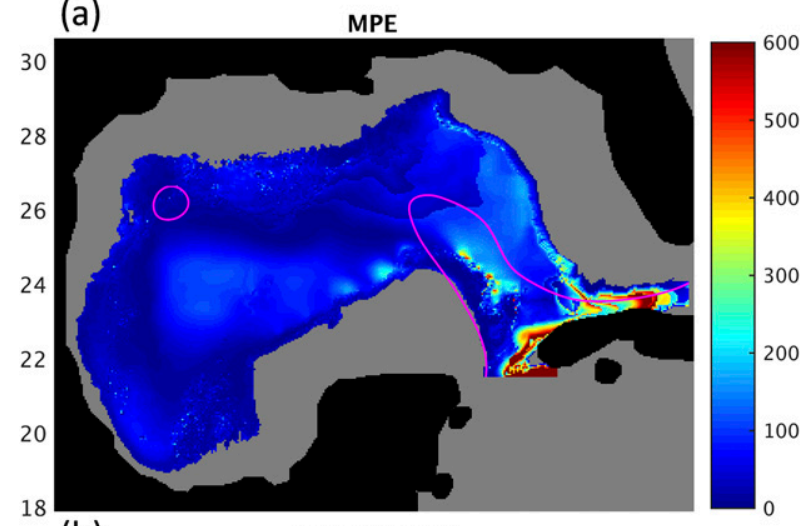

(b) MPE generation

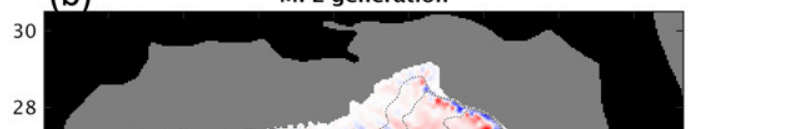

converted to MKE by the MPE $\rightarrow$ MKE conversion term and is related to baroclinic instabilities.

Comparing the MPE reservoir energy distribution using the whole computational domain (not shown) to the results with the domain limited to the GoM (Fig. 7a), it can be observed that the reference density has a noticeable effect on the result. The latter was also observed by $\mathrm{KC15}$ and Storch et al. (2012), and consequently further analysis of the MPE is not particularly meaningful. Although the reference density also has an effect on the conversion terms presented in Figs. $7 \mathrm{~b}$ and $7 \mathrm{c}$ this effect does not change the MPE energy generation and conversion physics as discussed before.

\section{c. Energy pathways}

As discussed in the previous sections, the energy transport between the upper- and lower-layer MPE and EPE is negligible and therefore will not be analyzed. The focus of this section is to understand the general energy transport of the EKE and MKE terms between the upper and lower layer and between the eastern and western part of the Gulf as a whole. A possible way to achieve this would be to divide the Gulf into many cross sections and then summarize the result for the whole Gulf. Nevertheless, we chose to use another method that consists of integrating the energy terms along the meridian direction (south-north) in a similar way as is usually done in a vertical direction (depth integrated).

\section{1) EKE SOURCES AND PATHWAYS}

The ROMS vertical coordinate is defined to be negative when pointing from the surface to the ocean floor. Accordingly, the vertical eddy $\mathrm{PW}^{\prime}$ flux must be negative to transfer energy into the deep GoM. To achieve the negative vertical $\mathrm{PW}^{\prime}$ flux the values of eddy pressure $p^{\prime}$ and eddy vertical velocity $w^{\prime}$ must have opposite signs. In the simulations, cyclonic eddies predominantly exhibit negative $p^{\prime}$ values, because their pressure is smaller than the average pressure, and they display positive $w^{\prime}$ values, which means that the vertical velocities are higher than the average $\bar{w}$ (pointing upward or less downward). In the case of deep anticyclonic eddies, the opposite situation is observed; $p^{\prime}$ values are positive, and $w^{\prime}$ values are negative.

The article by Zhai and Marshall (2013) was the main inspiration for the analysis of the energy pathways, which proposed that the vertical eddy energy fluxes $\overline{p^{\prime} w^{\prime}}$ exist due to mismatches between the depths at which the eddy energy is generated through baroclinic instability $(\mathrm{EPE} \rightarrow \mathrm{EKE})$ and the horizontal eddy energy fluxes that disperse the eddy energy. They correctly assume that the EPE $\rightarrow$ EKE term is the main source for the

Similarly, as in the EPE case, all the terms in Eq. (17) except for the turbulent diffusion term $(\bar{S} \& \overline{\mathrm{TD}})$ and MKE $\rightarrow$ MPE term produce negligible results. This means that the MPE energy generated by the turbulent diffusion and stored in the mean stratification is

FIG. 7. Comparison of the horizontal distributions of the deep ayer depth-integrated MPE reservoir $\left(\mathrm{J} \mathrm{m}^{-2}\right)$ and the dom intense MPE generation colors represent values higher or equal to $0.01 \mathrm{~W} \mathrm{~m}^{-2}$, while the most intense MPE dissipation colors epresent values smaller or equal to $-0.01 \mathrm{~W} \mathrm{~m}^{-2}$. The purple of the LC, and the bathymetry contours are spaced at -2000 $-2500,-3000$, and $-3500 \mathrm{~m}$. 
eddy energy generation as their results show that integrating over the whole North Atlantic Ocean, the rate of APE extracted by baroclinic instability (EPE $\rightarrow$ EKE) amounts to approximately $80 \%$ of the all wind energy input. This appears to be a valid approach on the large scale where the wind is the principal source of energy but is not adequate to be used in the case of the GoM where in fact, other terms in Eqs. (14) and (15), would prove to have a greater influence on the vertical energy flux than the baroclinic term.

As already mentioned in this work, a divergence of the vertical PW energy in the upper layer must result in a convergence of vertical PW energy in the lower layer. Therefore, to understand the vertical transport of energy, it is necessary to understand the intricacies between the divergence of vertical PW and the other terms in Eqs. (14) and (15). These equations represent a budget, where the LFS is approximately zero, and the terms on the RHS are in balance. This means that the outflow of energy with one term must be balanced by the inflow of energy by the other terms. In this context, we were interested in finding out which are the terms causing the outflow of energy by the divergence of the vertical PW in the upper layer, which results in a convergence of vertical PW energy in the lower layer.

Figure 8 presents the basin-averaged vertical profiles of the terms in Eq. (14) subsampled over the cells that contain only the divergent vertical $\mathrm{PW}^{\prime}$, which has a central role in transferring the EKE from the upper layer to the lower layer. We can observe that the most negative values of vertical $\mathrm{PW}^{\prime}$ are predominantly balanced by the horizontal $\mathrm{PW}^{\prime}+F^{\prime} \& \mathrm{TD}^{\prime}$ term and that the horizontal redistribution and diffusion of energy has a dominant role in pushing the energy in the vertical direction. Moreover, we can note that the baroclinic term $(\mathrm{EPE} \rightarrow \mathrm{EKE})$ and the barotropic term $(\mathrm{MKE} \rightarrow \mathrm{EKE})$ are a range of magnitude smaller, while the baroclinic term still presents higher values than the barotropic term. The discovery of the primary source of the vertical energy flux represents an important contribution of this work.

The EKE reservoir (Fig. 9a) illustrates higher values of energy in the deep layer in respect to MKE reservoir (Fig. 11a) as previously observed in the energy diagram (Fig. 3). The deep EKE is noticeably stronger in the eastern part of the Gulf under the mean location of the LC approximately from $88^{\circ}$ to $85^{\circ} \mathrm{W}$, as previously observed in Fig. 4b. Although a beam of energy reaching the floor can also be observed in the western part of the Gulf.

Most of the conversion term related to the baroclinic instabilities (EPE $\rightarrow$ EKE) is confined to the upper $1000 \mathrm{~m}$, while most of the energy conversion between the mean and the eddy flow related to barotropic

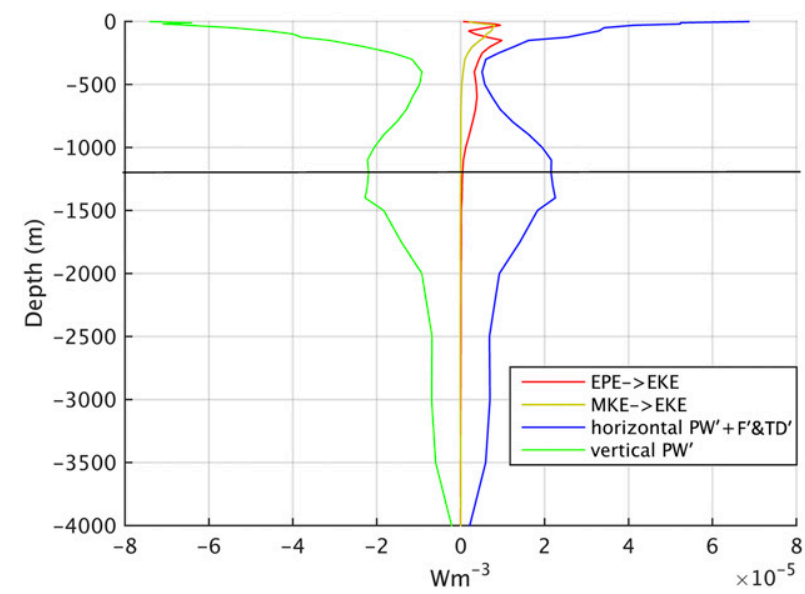

FIG. 8. Basin-averaged vertical profiles of the dominant terms in EKE budget Eq. (14) in the GoM. The terms are subsampled over the grid points with a diverging vertical $\mathrm{PW}^{\prime}\left(\partial \overline{w^{\prime} p^{\prime}} / \partial z>0\right)$, which has a central role in transferring the EKE from the upper layer to the deep layer. It is important to note the most negative values of vertical $\mathrm{PW}^{\prime}$ are predominantly balanced by the horizontal $\mathrm{PW}^{\prime}+F^{\prime} \& \mathrm{TD}^{\prime}$ term, meaning that the horizontal redistribution and diffusion of energy has a dominant role in pushing the energy in the vertical direction, while the baroclinic term $(\mathrm{EPE} \rightarrow \mathrm{EKE})$ and the barotropic term $(\mathrm{MKE} \rightarrow \mathrm{EKE})$ are a range of magnitude smaller.

instabilities (MKE $\rightarrow$ EKE) is confined to the upper $500 \mathrm{~m}$ (Fig. 9c). Both conversion terms are especially strong and penetrate deeper into the water column in the eastern part of the Gulf under the LC. The dashed black squares in Figs. 9b, 9c, and 9e denote the area of the Gulf where the vertical energy transfer by the vertical eddy $\mathrm{PW}^{\prime}$ is particularly noticeable and will be further discussed. High values of vertical eddy $\mathrm{PW}^{\prime}$ can be observed in the lower part of the square (below $1000 \mathrm{~m}$ ) and a colorful representation of all the other terms in its upper part (Fig. 9c), supporting the finding that in the Gulf the EPE $\rightarrow$ EKE term is not the dominant term causing vertical energy transport.

Although Fig. 9c indicates that the EPE $\rightarrow$ EKE term is not the dominant term in the area of pronounced vertical transport, it does not provide the information from exactly which terms originate in the vertical $\mathrm{PW}^{\prime}$ in the deep GoM. To obtain this information we have to compare the distribution of the energy terms in the upper layer of the EKE generation Fig. 9c to the area covered by the vertical $\mathrm{PW}^{\prime}$ in the upper layer of the EKE dissipation Fig. 9e. It can be observed that vertical eddy $\mathrm{PW}^{\prime}$ (green color area in Fig. 9e) covers mostly the area of the EPE $\rightarrow$ EKE term (Fig. 9c) and the area near the surface covered by the horizontal $\mathrm{PW}^{\prime}+F^{\prime} \& \mathrm{TD}^{\prime}$ term (Fig. 9c). Although the contribution of the $\mathrm{EPE} \rightarrow$ EKE term (Fig. 9c) to the dissipation (divergence) of vertical PW' (Fig. 9e) appear to be higher than the other energy terms, its basin averaged values are 

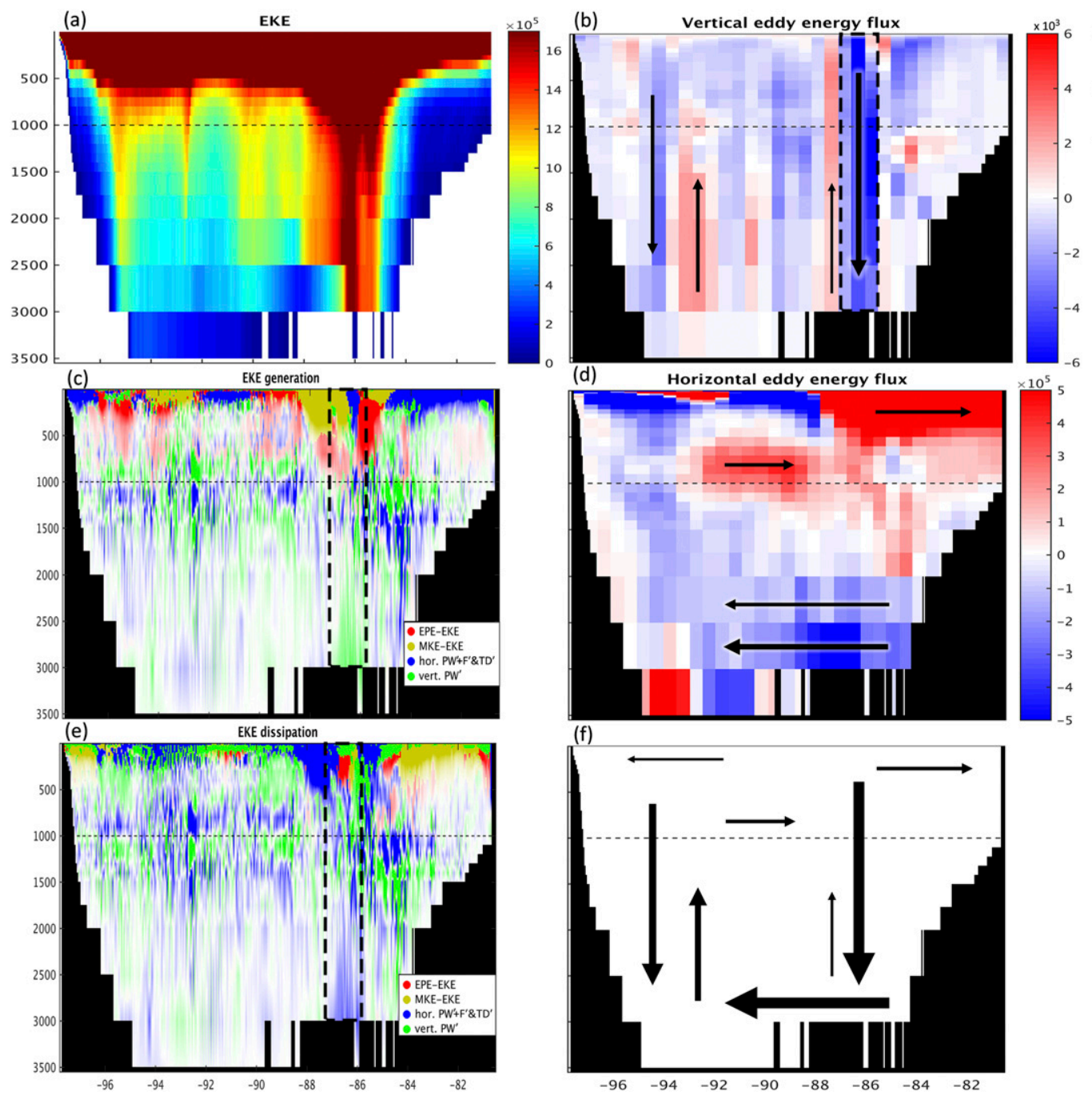

FIG. 9. Comparison of the vertical distributions along the meridian integrated (a) EKE reservoir $\left(\mathrm{J} \mathrm{m}^{-2}\right)$, (b) vertical eddy energy flux $\overline{w^{\prime} p^{\prime}}\left(\mathrm{W} \mathrm{m}^{-2}\right)$, (c) dominant EKE generation terms, (d) horizontal (east-west) eddy energy flux $\overline{u^{\prime} p^{\prime}}\left(\mathrm{W} \mathrm{m}^{-2}\right)$, (e) dominant EKE dissipation terms, and (f) schematic of the horizontal and vertical eddy energy flux. The most intense EKE generation colors represent values higher or equal to $10 \mathrm{~W} \mathrm{~m}^{-2}$, while the most intense EKE dissipation colors represent values smaller or equal to $-10 \mathrm{~W} \mathrm{~m}^{-2}$. The black vectors symbolize the direction of the energy transfer, while the dashed black square delimits the region with strong downward EKE flux, further analyzed in the text.

a range of magnitude smaller than the contribution of the horizontal $\mathrm{PW}^{\prime}+F^{\prime} \& \mathrm{TD}^{\prime}$ term, especially up to a depth of $250 \mathrm{~m}$ (Fig. 8). Finally, the energy transported from the upper layer by the vertical $\mathrm{PW}^{\prime}$ is redistributed and dissipated in the lower layer by the horizontal $\mathrm{PW}^{\prime}+F^{\prime} \& \mathrm{TD}^{\prime}$ term (Fig. 9e).

As expected, strong vertical energy flux $\overline{p^{\prime} w^{\prime}}$ in the eastern Gulf (Fig. 9b) is transferring energy to the deep layer where the EKE generation due to vertical $\mathrm{PW}^{\prime}$ presents its highest values (Fig. 9c). Just east of this beam of energy is a narrow vertical eddy energy flux pointing upward at approximately $88^{\circ} \mathrm{W}$ located under the tip of the LC, most likely related to the narrowing of the bathymetry in this region. Another noticeable beam of vertical eddy energy flux is located in the western part of the Gulf from $94^{\circ}$ to $92^{\circ} \mathrm{W}$, which is roughly the location of the rough bathymetry. On the far western part of the Gulf (west of $94^{\circ} \mathrm{W}$ ), it is possible to observe another beam of energy pointing downward, possibly related to the LCEs interaction with the western bathymetry.

Figure $9 \mathrm{~d}$ presents the horizontal (east-west) eddy energy flux $\overline{u^{\prime} p^{\prime}}$, where strong westward energy transport can be observed below a depth of $2000 \mathrm{~m}$. This result is expected because the Gulf under the depth of the 
Yucatan sill $(1900 \mathrm{~m})$ represents a closed region, and a horizontal eddy energy flux is used to redistribute the convergence of the vertical eddy $\mathrm{PW}^{\prime}$ across the Gulf. Additionally, because the amount of vertical $\mathrm{PW}^{\prime}$ on the eastern side of the Gulf surpasses the one on the western side the result is a westward transport of eddy energy. As mentioned, the horizontal westward transport gets stronger below $2000 \mathrm{~m}$, when also the amount the EKE generated by the vertical $\mathrm{PW}^{\prime}$ starts to increase more noticeably (Fig. 9c). The convergence of the vertical $\mathrm{PW}^{\prime}$ starts to increase toward the bottom because the vertical eddy energy flux is interrupted by the GoM floor, and the energy starts to accumulate and disperse in the horizontal direction. The present understanding of the GoM horizontal and vertical eddy energy flux is summarized in a schematic drawing (Fig. 9f).

\section{2) MKE SOURCES AND PATHWAYS}

Figure 10 shows that also in the case of MKE the dominant term pushing the energy downward is the horizontal $\overline{\mathrm{PW}}+\bar{F} \& \overline{\mathrm{TD}}$ term and that the baroclinic term is an order of magnitude smaller, while the barotropic term (EKE $\rightarrow$ MKE) does not contribute to the vertical energy transport.

The maintaining of the MKE reservoir by the different contributions of the dominant terms can be observed by the comparison of Figs. 11a and 11c, respectively. In contrast to the EKE reservoir (Fig. 9a) where the energy beams to the deep layer are gradually decreasing from the surface to the seafloor, the MKE reservoir presents an increase of MKE energy most noticeably between 1500- and 2500-m depth in the eastern side of the Gulf and below $2000 \mathrm{~m}$ in its western side. The increase of MKE in the eastern side is related to the generation of MPE by the turbulent diffusion over the rough bathymetry and steep slopes (Fig. 7b) and is then converted to MKE by the MPE $\rightarrow$ MKE conversion term (Figs. 7c and 11c). On the other hand, the increase of MKE in the western side below 2000-m depth is related to the vertical $\overline{\mathrm{PW}}$ (Fig. 11c). Most of the conversion term related to the baroclinic instabilities (MPE $\rightarrow$ MKE) is confined to the upper $1000 \mathrm{~m}$, while most of the energy conversion related to barotropic instabilities $(\mathrm{EKE} \rightarrow \mathrm{MKE}$ ) is confined to the upper $250 \mathrm{~m}$ (Fig. 11c). Both conversion terms penetrate deeper into the water column in the eastern part of the Gulf. Observing the distribution of the dominant MKE generation terms (Fig. 11c), it is not clearly visible which term in the upper layer is dominant in generating the vertical $\overline{\mathrm{PW}}$ in the lower layer. The same was true also for the vertical $\mathrm{PW}^{\prime}$ (Fig. 9c) as discussed in the previous chapter. The core of the energy released from the mean flow to generate eddies (EKE $\rightarrow$ MKE) can be observed from

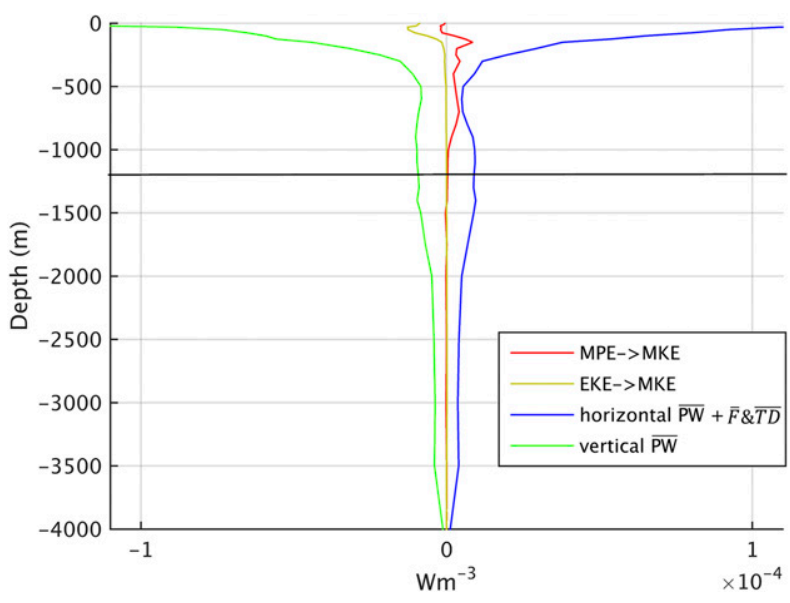

FIG. 10. Basin-averaged vertical profiles of the dominant terms in the MKE budget equation (15) in the GoM. The terms are subsampled over the grid points with a diverging vertical $\overline{\mathrm{PW}}\left(\partial \bar{w} \overline{p_{a}} / \partial z>0\right)$, which has a central role in transferring the MKE from the upper layer to the deep layer. It is important to note the most negative values of vertical $\overline{\mathrm{PW}}$ are predominantly balanced by the horizontal $\overline{\mathrm{PW}}+\bar{F} \& \overline{\mathrm{TD}}$ term, meaning that the horizontal redistribution and diffusion of energy has a dominant role in pushing the energy in the vertical direction, while the baroclinic term (MPE $\rightarrow$ MKE) and the barotropic term (EKE $\rightarrow$ MKE) are a range of magnitude smaller.

$88^{\circ}$ to $86^{\circ} \mathrm{W}$, roughly the location of the LC (Fig. 5e) and can exceed the depth of $500 \mathrm{~m}$ (Fig. 11e). It is important to note that the distribution of the mean energy flux (Fig. 11b) is sensitive to the choice of the computational domain and should be considered as an approximation. Nevertheless, the general energy pathways remain consistent.

Figure 11d presents the horizontal (east-west) mean energy flux, where the same strong westward energy transport can be observed below 2000-m depth, as observed in the case of the horizontal eddy energy pathways (Fig. 9d). As in the aforementioned case, this result is expected because the amount of the vertical $\overline{\mathrm{PW}}$ on the eastern side of the Gulf surpasses the one on the western side, and the horizontal mean energy flux redistributes the convergence of the vertical $\overline{\mathrm{PW}}$ across the Gulf, resulting in a westward transport of energy (Fig. 11d). Finally, the schematic of the GoM horizontal and vertical mean energy flux is presented in Fig. 11f.

\section{Summary and conclusions}

Based on the outputs of the ROMS ocean model, the energetics of the deep GoM have been investigated in this study. The theoretical framework for the analysis is based on the energy equations for the time-mean and time-varying flow ( $\mathrm{KC15}$ ), where some of the terms were additionally split into their horizontal and vertical components to monitor the energy pathways as introduced by Zhai and Marshall (2013). Using these equations, we 

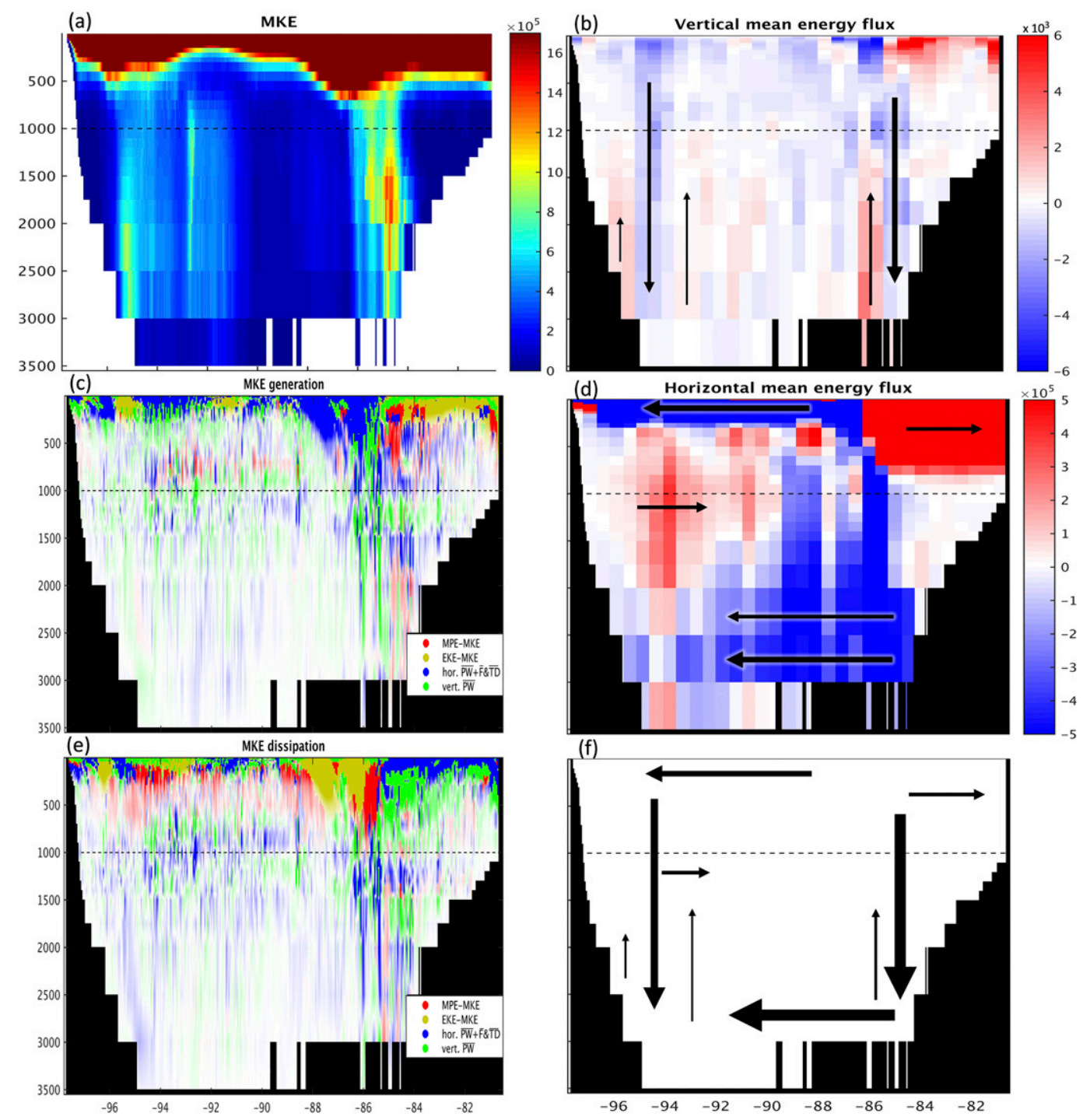

FIG. 11. Comparison of the vertical distributions along the meridian integrated (a) MKE reservoir $\left(\mathrm{J} \mathrm{m}^{-2}\right)$, (b) vertical mean energy flux $\bar{w} \overline{p_{a}}\left(\mathrm{~W} \mathrm{~m}^{-2}\right)$, (c) dominant MKE generation terms, (d) horizontal (east-west) mean energy flux $\bar{u} \overline{p_{a}}\left(\mathrm{~W} \mathrm{~m}^{-2}\right)$, (e) dominant MKE dissipation terms, and (f) schematic of the horizontal and vertical mean energy flux. The most intense MKE generation colors represent values higher or equal to $10 \mathrm{~W} \mathrm{~m}^{-2}$, while the most intense MKE dissipation colors represent values smaller or equal to $-10 \mathrm{~W} \mathrm{~m}^{-2}$. The black vectors symbolize the direction of the energy transfer.

examined the energy cycle, eddy-mean flow interactions, and energy pathways focusing on the deep Gulf.

The evaluation of the deep GoM energy cycle is an important contribution of this article and provided the answer to our main research question about how the energy driving the deep GoM circulation is sustained. We find that the KE of the deep circulation is predominantly maintained $(75 \%)$ by the energy redistribution from the upper to the lower layer by the vertical PW, followed by a considerable amount $(19 \%)$ by the conversion of energy from the potential to kinetic energy related to baroclinic instabilities and the rest $(\sim 6 \%)$ by the horizontal PW. Finally, it is interesting to note that in the upper layer, the mean circulation is a generator of eddies while in the deep layer, the eddies drive the mean circulation. These results appear to suggest that, in the GoM, accurately simulating the current in the upper layer is necessary to also successfully simulate the circulation in the deep layer.

A more complete understanding of the spatial distribution of the terms from the energy equations was achieved by analyzing their depth-integrated values, 
separately, for the upper and the deep layer. To avoid an overwhelming number of figures, which is usually associated with the energetics analysis, and to determine the energy terms with a dominant role in maintaining the deep circulation, a novel approach was implemented to analyze the result. The new approach consisted of analyzing all the terms in a specific energy equation at the same time in only two figures, the first figure focusing on the energy generation and the second figure on the energy dissipation. The benefit of this approach is the density of information about the dominant energy generation and dissipation terms. The disadvantage is that information was lost regarding the nondominant terms, especially when there exist generation (dissipation) terms having comparable strengths. Nevertheless, we believe that the advantages outweigh the disadvantages and that the method could be used in the future for energetics analyses exploring the dominant terms.

The EKE generation and dissipation terms in the deep layer are roughly evenly distributed across the Gulf, while the most intense MKE generation and dissipation terms are concentrated along its boundary where the strongest boundary currents are located. The results show a strong eddy energy transfer from the upper to the deep layer by the vertical $\mathrm{PW}^{\prime}$ below the area delimited by the mean LC (17-cm SSH contour) position. On the other hand, the highest values of mean vertical $\overline{\mathrm{PW}}$ can be observed in the areas of the rough bathymetry and in the area of the steep slopes under the LC. The eddies tend to accelerate the mean flow along the northern part of the Gulf, where they collide with the continental slope, and in the area under the LC.

In contrast to the analysis of the EKE and MPE reservoirs, the interpretation of the EPE and MPE results is rather simple because there are only two dominant terms affecting each energy reservoir, the turbulent diffusion terms $\left(S^{\prime} \& \mathrm{TD}^{\prime}\right.$ and $\left.\bar{S} \& \overline{\mathrm{TD}}\right)$ and the terms related to baroclinic instabilities (EPE $\rightarrow$ EKE and $\mathrm{MPE} \rightarrow \mathrm{MKE}$ ). The turbulent diffusion terms are generating MPE and EPE in the areas of rough bathymetry and steep slopes where the subgrid diffusion is destabilizing the stratification resulting in an increase of APE. The APE is stored in the tilted isopycnals and then converted to $\mathrm{KE}$ by the terms related to baroclinic instabilities.

Another important part of this research was to understand the general energy transport pathways between the upper and lower layers and the eastern and western parts of the Gulf. The energy transport between the upper and lower layer MPE and EPE was found to be negligible, and therefore only the EKE and MKE energy pathways were analyzed. The latter showed very similar properties of the general energy pathways. Energy is transferred downward in the eastern and the western part of the Gulf and upward in the deep centralwestern part. Because the downward transport of energy underneath the LC in the eastern GoM is greater than in the western GoM, a strong westward energy transport can be observed below 2000-m depth. Furthermore, we observed that the primary source for the vertical energy flux is the horizontal PW and the turbulent diffusion in the upper layer. This represents a difference between our work and the work of Zhai and Marshall (2013) and highlights an important conclusion of this work. This conclusion is that the baroclinic term is not, by default, the primary source of the vertical eddy energy flux and that further analysis is necessary to determine which are the dominant terms in the energy equations generating the energy transport in the vertical direction.

Although great care was taken to ensure the highest accuracy of the results, there is still room to reduce their computational error. The error arises with the interpolation of the ROMS terrain-following coordinates onto the constant depth coordinates. A better approach might be to implement the energy equations into ROMS and interpolate the final results. Similarly, other authors using models with terrain-following vertical coordinates (KC15; Stepanov 2018) did not report using energy equations adjusted for the sigma coordinates from what we are deducing that they probably used interpolations in their analysis. Accordingly, we are assuming that the interpolated results are sufficiently accurate, and therefore considered appropriate in the energetics analysis.

This paper presents a first attempt in describing a three-dimensional picture of the deep GoM energy cycle, eddy-mean flow interactions, and the energy pathways. These results could present a benchmark for a future model and observational studies of the deep Gulf. Furthermore, the methods described in this work can be applied to other regional or global oceans to study the energy cycle and pathways between the surface and the bottom waters. It should be noted that more studies are required to clarify the triggering mechanism of the downward energy transfer, and more work is required to fully understand the process related to the generation of EKE from EPE in the case of nontraditional baroclinic instability energy pathway.

Acknowledgments. The study was funded by the National Academies of Sciences, Gulf Research Program (NASGRP) under Grant Number 200006422 and by the National Council of Science and Technology of Mexico, Mexican Ministry of Energy, Hydrocarbon Trust, as part of the Gulf of Mexico Research Consortium (CIGoM), project 201441. The authors wish to thank Matt K. Gough who reviewed and provided useful comments on the manuscript. 


\section{APPENDIX}

\section{Divergence of Vertical PW in the Upper Layer of the Gulf Causes a Convergence of Vertical PW in the Lower Layer}

In this section, we provide evidence that a divergence of vertical PW in the upper layer of the GoM must result in a convergence of vertical PW energy in the lower layer and vice versa. The evidence is presented only for the eddy vertical $\mathrm{PW}^{\prime}$ because the proof for the mean vertical $\overline{\mathrm{PW}}$ follows the same lines.

Figure A1 represents a sketch of the water column control volume consisting of two subvolumes. The lower volume $V_{1}$ is bounded by the surface $S_{1}$ while the upper volume $V_{2}$ is bounded by the surface $S_{2}$. Both surfaces are oriented outward, where $\mathbf{n}$ is the positive normal to the surface. The upper surface of the control volume $\left(S_{1 \mathrm{~m}}\right)$ is placed at 1-m depth (rather than on the sea surface) because by following the definition of the divergence theorem the vector field has to be defined inside the control volume, which above the 1-m depth is not always the case due to the free-surface fluctuations. The surface $S_{1000 \mathrm{~m}}$ represents a common face between the two subvolumes.

In the proof of the divergence theorem, it is demonstrated that a surface integral of the component of a vector field in $\mathbf{k}$ direction (Fig. A1) is equal to the volume integral of the partial derivative with respect to the variable $z$ (Fig. A1) of that component over the region inside the surface. Accordingly, the following equations can be written for the eddy vertical $\mathrm{PW}^{\prime}$ separately for each subvolume:

$$
\begin{aligned}
& \iiint_{V_{2}} \frac{\partial \overline{w^{\prime} p^{\prime}}}{\partial z} d V=\iint_{S_{2}} \overline{w^{\prime} p^{\prime}}(\mathbf{k} \cdot \mathbf{n}) d S, \\
& \iiint_{V_{1}} \frac{\partial \overline{w^{\prime} p^{\prime}}}{\partial z} d V=\iint_{S_{1}} \overline{w^{\prime} p^{\prime}}(\mathbf{k} \cdot \mathbf{n}) d S .
\end{aligned}
$$

The RHS of Eq. (A1) is further evaluated with the $\overline{w^{\prime} p^{\prime}}$ flux across the specific boundary surfaces

$$
\begin{aligned}
& \iiint_{V_{2}} \frac{\partial \overline{w^{\prime} p^{\prime}}}{\partial z} d V=-\iint_{S_{1000 \mathrm{~m}}} \overline{w^{\prime} p^{\prime}} d S+\underbrace{\iint_{S_{1 \mathrm{~m}}} \overline{w^{\prime} p^{\prime}} d S}_{\approx 0}, \quad(\mathrm{~A} 2) \\
& \iiint_{V_{1}} \frac{\partial \overline{w^{\prime} p^{\prime}}}{\partial z} d V=\iint_{S_{1000 \mathrm{~m}}} \overline{w^{\prime} p^{\prime}} d S+\underbrace{\iint_{S_{\mathrm{bottom}}} \overline{w^{\prime} p^{\prime}}(\mathbf{k} \cdot \mathbf{n}) d S}_{=0} .
\end{aligned}
$$

The second term on the RHS in Eq. (A2) is neglected because our data show that the $\overline{w^{\prime} p^{\prime}}$ flux across the surface

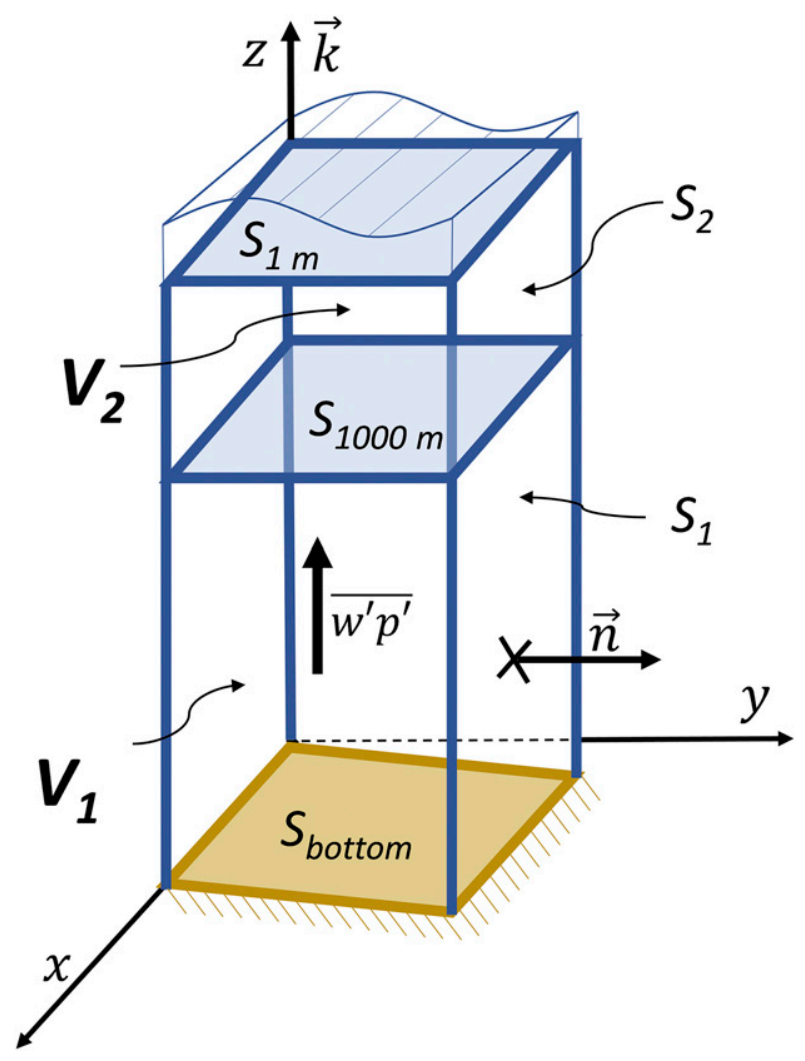

FIG. A1. Water column control volume used for the explanation of the vertical PW energy transfer.

$S_{1 \mathrm{~m}}$ at 1-m depth is a range of magnitude smaller than the $\overline{w^{\prime} p^{\prime}}$ flux across the $1000-\mathrm{m}$ depth. This is somewhat expected since the vertical velocity $w$ at 1-m depth represents the slow variations of the free-surface high. On the other hand, the second term on the RHS in Eq. (A3) is equal to zero for the following reasons:

1) In fluid mechanics, we generally assume that in a thin (few millimeters) layer of fluid in contact with a solid boundary the normal and the parallel velocity to a boundary are equal to zero from which follows that the vertical velocity $w$ at the bottom is equal to zero no matter the slope of the bottom and consequently, also the value of the related term $\overline{w^{\prime} p^{\prime}}$ at the bottom is equal to zero.

2) In the ROMS simulations, a quadratic function of velocity (Cushman-Roisin and Beckers 2011) was used to parameterize the bottom friction with a nondimensional constant bottom drag coefficient $\mathrm{Cd}=0.003$. The estimation of the drag coefficient (Lueck and Lu 1997) assumes a logarithmic velocity profile near the sea bed (Cushman-Roisin and Beckers 2011), where the parallel velocity in contact with a boundary is zero. Thus, also in the simulations the vertical velocity $w$ at the bottom and the related term in Eq. (A3) are zero no matter the slope of the sea bed. 
The last step in the proof is to add Eqs. (A2) and (A3) to obtain

$$
\iiint_{V_{1}} \frac{\partial \overline{w^{\prime} p^{\prime}}}{\partial z} d V+\iiint_{V_{2}} \frac{\partial \overline{w^{\prime} p^{\prime}}}{\partial z} \approx 0
$$

Equation (A4) shows that the divergence of the vertical eddy $\mathrm{PW}^{\prime}$ in the upper volume must result in the convergence of vertical eddy $\mathrm{PW}^{\prime}$ in the lower volume and this is how the energy is transferred from the upper layer to the lower layer and the other way around.

\section{REFERENCES}

Alvera-Azcárate, A., A. Barth, and R. H. Weisberg, 2009: The surface circulation of the Caribbean Sea and the Gulf of Mexico as inferred from satellite altimetry. J. Phys. Oceanogr., 39, 640-657, https://doi.org/10.1175/2008JPO3765.1.

Beckmann, A., C. W. Böning, B. Brügge, and D. Stammer, 1994: On the generation and role of eddy variability in the central North Atlantic Ocean. J. Geophys. Res., 99, 20381-20391, https://doi.org/10.1029/94JC01654.

Biggs, D. C., G. S. Fargion, P. Hamilton, and R. R. Leben, 1996: Cleavage of a Gulf of Mexico loop current eddy by a deep water cyclone. J. Geophys. Res., 101, 20 629-20 641, https:// doi.org/10.1029/96JC01078.

Cardona, Y., and A. Bracco, 2016: Predictability of mesoscale circulation throughout the water column in the Gulf of Mexico. Deep-Sea Res. II, 129, 332-349, https://doi.org/10.1016/ j.dsr2.2014.01.008.

Chang, Y.-L., and L.-Y. Oey, 2011: Loop current cycle: Coupled response of the loop current with deep flows. J. Phys. Oceanogr., 41, 458-471, https://doi.org/1.1175/2010JPO4479.1.

Chen, R., G. R. Flierl, and C. Wunsch, 2014: A description of local and nonlocal eddyMean flow interaction in a global eddypermitting state estimate. J. Phys. Oceanogr., 44, 2336-2352, https://doi.org/10.1175/JPO-D-14-0009.1.

- A. F. Thompson, and G. R. Flierl, 2016: Time-dependent eddy-mean energy diagrams and their application to the ocean. J. Phys. Oceanogr., 46, 2827-2850, https://doi.org/ 10.1175/JPO-D-16-0012.1.

Cushman-Roisin, B., and J.-M. Beckers, 2011: Introduction to Geophysical Fluid Dynamics: Physical and Numerical Aspects. Academic Press, 828 pp.

DeHaan, C. J., and W. Sturges, 2005: Deep cyclonic circulation in the Gulf of Mexico. J. Phys. Oceanogr., 35, 1801-1812, https:// doi.org/10.1175/JPO2790.1.

Donohue, K. A., D. R. Watts, P. Hamilton, R. Leben, and M. Kennelly, 2016: Loop current eddy formation and baroclinic instability. Dyn. Atmos. Oceans, 76, 195-216, https:// doi.org/10.1016/j.dynatmoce.2016.01.004.

Eden, C., and C. Böning, 2002: Sources of eddy kinetic energy in the Labrador Sea. J. Phys. Oceanogr., 32, 3346-3363, https://doi.org/ 10.1175/1520-0485(2002)032<3346:SOEKEI >2.0.CO;2.

Ferrari, R., and C. Wunsch, 2009: Ocean circulation kinetic energy: Reservoirs, sources, and sinks. Annu. Rev. Fluid. Mech., 41, 253282, https://doi.org/10.1146/annurev.fluid.40.111406.102139.

Flury, J., and R. Rummel, 2007: Future Satellite Gravimetry and Earth Dynamics. Springer Science and Business Media, 164 pp.

Galperin, B., L. H. Kantha, S. Hassid, and A. Rosati, 1988: A quasi-equilibrium turbulent energy model for geophysical flows. J. Atmos. Sci., 45, 55-62, https://doi.org/10.1175/15200469(1988)045<0055:AQETEM>2.0.CO;2.

Garcia-Jove, M., J. Sheinbaum, and J. Jouanno, 2016: Sensitivity of loop current metrics and eddy detachments to different model configurations: The impact of topography and Caribbean perturbations. Atmósfera, 29, 235-265, https://doi.org/10.20937/ ATM.2016.29.03.05.

Gill, A. E., 1982: Atmosphere-Ocean Dynamics. Academic Press, $662 \mathrm{pp}$.

Hamilton, P., 2009: Topographic Rossby waves in the Gulf of Mexico. Prog. Oceanogr., 82, 1-31, https://doi.org/10.1016/ j.pocean.2009.04.019.

_ , and A. LugoFernandez, 2001: Observations of high speed deep currents in the northern Gulf of Mexico. Geophys. Res. Lett., 28, 2867-2870, https://doi.org/10.1029/2001GL013039.

— G. Fargion, and D. Biggs, 1999: Loop current eddy paths in the western Gulf of Mexico. J. Phys. Oceanogr., 29, 1180-1207, https://doi.org/10.1175/1520-0485(1999)029<1180:LCEPIT> 2.0.CO;2.

— A. Bower, H. Furey, R. Leben, and P. Pérez-Brunius, 2016: Deep circulation in the Gulf of Mexico: A Lagrangian study. OCS Study BOEM 2016-081, 289 pp., https://www.boem.gov/ Gulf-of-Mexico-OCS-Region-Publications-G/\#GULF\%20OF\% 20MEXICO.

Harrison, D. E., and A. R. Robinson, 1978: Energy analysis of open regions of turbulent flows-Mean eddy energetics of a numerical ocean circulation experiment. Dyn. Atmos. Oceans, 2 , 185-211, https://doi.org/10.1016/0377-0265(78)90009-X.

Holland, W. R., D. E. Harrison, and A. J. Semtner, 1983: Eddyresolving numerical models of large-scale ocean circulation. Eddies in Marine Science, A. R. Robinson, Ed., Springer, 379-403.

Kang, D., and E. N. Curchitser, 2015: Energetics of eddy-mean flow interactions in the Gulf Stream region. J. Phys. Oceanogr., 45, 1103-1120, https://doi.org/10.1175/JPO-D-14-0200.1.

— , and — 2017: On the evaluation of seasonal variability of the ocean kinetic energy. J. Phys. Oceanogr., 47, 1675-1683, https://doi.org/10.1175/JPO-D-17-0063.1.

Leben, R. R., 2013: Altimeter-derived loop current metrics. Circulation in the Gulf of Mexico: Observations and Models, Geophys. Monogr., Vol. 161, Amer. Geophys. Union, 181-201.

Lee, H.-C., and G. L. Mellor, 2003: Numerical simulation of the Gulf Stream system: The loop current and the deep circulation. J. Geophys. Res., 108, 3043, https://doi.org/10.1029/2001JC001074.

Levitus, S., 1982: Climatological Atlas of the World Ocean. National Oceanic and Atmospheric Administration, 173 pp.

Lorenz, E. N., 1955: Available potential energy and the maintenance of the general circulation. Tellus, 7, 157-167, https:// doi.org/10.3402/tellusa.v7i2.8796.

Lueck, R. G., and Y. Lu, 1997: The logarithmic layer in a tidal channel. Cont. Shelf Res., 17, 1785-1801, https://doi.org/ 10.1016/S0278-4343(97)00049-6.

Maslo, A., J. M. A. C. de Souza, F. Andrade-Canto, and J. R. Outerelo, 2020: Connectivity of deep waters in the Gulf of Mexico. J. Mar. Syst., 203, 103267, https://doi.org/10.1016/ J.JMARSYS.2019.103267.

Mellor, G. L., and T. Yamada, 1982: Development of a turbulence closure model for geophysical fluid problems. Rev. Geophys., 20, 851-875, https://doi.org/10.1029/RG020i004p00851.

Oey, L., and H. Lee, 2002: Deep eddy energy and topographic Rossby waves in the Gulf of Mexico. J. Phys. Oceanogr., 32, 3499-3527, https://doi.org/10.1175/1520-0485(2002)032<3499: DEEATR $>2.0 . \mathrm{CO} ; 2$. 
, T. Ezer, and H. Lee, 2005: Loop current, rings and related circulation in the Gulf of Mexico: A review of numerical models and future challenges. Circulation in the Gulf of Mexico: Observations and Models, Geophys. Monogr., Vol. 161, Amer. Geophys. Union, 31-56.

Oey, L.-Y., 2008: Loop current and deep eddies. J. Phys. Oceanogr., 38, 1426-1449, https://doi.org/10.1175/2007JPO3818.1.

Olbers, D., J. Willebrand, and C. Eden, 2012: Ocean Dynamics. Springer Science and Business Media, 703 pp., https://doi.org/ 10.1007/978-3-642-23450-7.

Orlanski, I., and M. D. Cox, 1972: Baroclinic instability in ocean currents. Geophys. Fluid Dyn., 4, 297-332, https://oi.org/ 10.1080/03091927208236102.

Pérez-Brunius, P., H. Furey, A. Bower, P. Hamilton, J. Candela, P. García-Carrillo, and R. Leben, 2018: Dominant circulation patterns of the deep Gulf of Mexico. J. Phys. Oceanogr., 48, 511-529, https://doi.org/10.1175/JPO-D-17-0140.1.

Shchepetkin, A. F., and J. C. McWilliams, 2005: The Regional Oceanic Modeling System (ROMS): A split-explicit, free-surface, topography-following-coordinate oceanic model. Ocean Modell., 9, 347-404, https://doi.org/10.1016/j.ocemod.2004.08.002.

Smagorinsky, J., 1963: General circulation experiments with the primitive equations. Mon. Wea. Rev., 91, 99-164, https://doi.org/10.1175/ 1520-0493(1963)091<0099:GCEWTP>2.3.CO;2.

Stepanov, D. V., 2018: Eddy energy sources and mesoscale eddies in the Sea of Okhotsk. Ocean Dyn., 68, 825-845, https:// doi.org/10.1007/S10236-018-1167-3.
Storch, J.-S., and Coauthors, 2012: An estimate of the Lorenz energy cycle for the world ocean based on the STORM/NCEP simulation. J. Phys. Oceanogr., 42, 2185-2205, https://doi.org/ 10.1175/JPO-D-12-079.1.

Vukovich, F. M., 2007: Climatology of ocean features in the Gulf of Mexico using satellite remote sensing data. J. Phys. Oceanogr., 37, 689-707, https://doi.org/10.1175/JPO2989.1.

Weatherly, G. L., N. Wienders, and A. Romanou, 2005: Intermediate-depth circulation in the Gulf of Mexico estimated from direct measurements. Circulation in the Gulf of Mexico: Observations and Models, Geophys. Monogr., Vol. 161, Amer. Geophys. Union, 315-324.

Yang, H., L. Wu, H. Liu, and Y. Yu, 2013: Eddy energy sources and sinks in the South China Sea. J. Geophys. Res. Oceans, 118, 4716-4726, https://doi.org/10.1002/jgrc.20343.

Youngs, M. K., A. F. Thompson, A. Lazar, and K. J. Richards, 2017: ACC meanders, energy transfer, and mixed barotropicbaroclinic instability. J. Phys. Oceanogr., 47, 1291-1305, https://doi.org/10.1175/JPO-D-16-0160.1.

Zhai, X., and D. P. Marshall, 2013: Vertical eddy energy fluxes in the North Atlantic subtropical and subpolar gyres. J. Phys. Oceanogr., 43, 95-103, https://doi.org/10.1175/ JPO-D-12-021.1.

Zhan, P., A. C. Subramanian, F. Yao, A. R. Kartadikaria, D. Guo, and I. Hoteit, 2016: The eddy kinetic energy budget in the Red Sea. J. Geophys. Res. Oceans, 121, 4732-4747, https://doi.org/ 10.1002/2015JC011589. 